DAMTP/96-104

hep-th/9701093

\title{
EFFECTS OF D-INSTANTONS.
}

\author{
Michael B. Green! and Michael Gutperle
}

DAMTP, Silver Street, Cambridge CB3 9EW, UK.

\begin{abstract}
Scattering of fundamental states of type IIB supergravity and superstring theory is discussed at low orders in perturbation theory in the background of a D-instanton. The integration over fermionic zero modes in both the low energy supergravity and in the string theory leads to explicit nonperturbative terms in the effective action. These include a single instanton correction to the known tree-level and one-loop $R^{4}$ interactions. The 'spectrum' of multiply-charged D-instantons is deduced by T-duality in nine dimensions from multiply-wound world-lines of marginally-bound D-particles. This, and other clues, lead to a conjectured SL $(2, \mathrm{Z})$ completion of the $R^{4}$ terms which suggests that they are not renormalized by perturbative corrections in the zero-instanton sector beyond one loop. The string theory unit-charged D-instanton gives rise to point-like effects in fixed-angle scattering, raising unresolved issues concerning distance scales in superstring theory.
\end{abstract}

Keywords: D-instanton, IIB supergravity, duality, fermionic zero modes, pointlike scattering PACS: 11.25.-w, 11.25.Sq, 04.65.+e

\footnotetext{
${ }^{1}$ M.B.Green@damtp.cam.ac.uk

${ }^{2}$ M.Gutperle@damtp.cam.ac.uk
} 


\section{Introduction}

The dualities that relate superstring perturbation expansions (and M-theory) involve the interchange of fundamental string excitations and solitons. In compactified theories these symmetries relate the different states obtained by wrapping both the strings and their solitons around compact dimensions, but already in ten dimensions there are interesting inter-relationships between theories. Notably, the type IIB theory is self-dual in the sense that it transforms into itself under $S L(2, Z)$ transformations which act on the various species of solitons - the $S L(2, Z)$ multiplet of strings, the self-dual three-brane, the $S L(2, Z)$ multiplet of fivebranes and the seven-brane. However, the type IIB theory also possesses an instanton solution which couples to the Ramond-Ramond $(\mathrm{R} \otimes \mathrm{R})$ charge associated with the pseudoscalar, $\left.C^{(0)}\right]^{3}$ This leads to little-studied non-perturbative effects in the ten-dimensional theory - effects that are intimately related to the $S L(2, Z)$ symmetry of the theory.

After compactification there are other instantons, namely, BPS ' $(p+1)$-instantons' that arise from wrapping the $(p+1)$-dimensional euclidean world-volumes of $p$-branes around compact dimensions in a supersymmetric manner [1, 2, 3]. All such instantons, which are related to each other by T-duality, have infinite actions in the limit of flat ten-dimensional space-time apart from the D-instanton $(p=-1)$, which has a zero-dimensional world-volume. Such euclidean configurations may be treated in a unified manner, for example, using the formalism of [沟.

Although some properties of these $(p+1)$-instantons have been studied and their duality properties have been explored, relatively little is known about correlation functions in the instanton backgrounds and even less about the effect of the D-instanton on ten-dimensional type IIB theory. It would, for example, be interesting to study the $S L(2, Z)$ extension of the perturbative effective action, which might reveal connections with more fundamental underlying principles. It is particularly fascinating that D-instantons appear to give rise to point-like behaviour of short-distance correlation functions of fundamental strings. Indeed the point-like nature of Dirichlet boundary conditions has for some time been linked to power behaviour of fixed-angle scattering in the bosonic string theory [5, 6, 7]. This is in marked contrast to the exponential decrease of fixed-angle cross sections that is characteristic of conventional fundamental string processes $8,9,10$.

This paper is concerned with effects of D-instantons in IIB supergravity and superstring theory in ten uncompactified dimensions. Much of the analysis also applies to any of the $(p+1)$-instantons that arise in compactifications to lower dimensions. We will begin by reviewing the D-instanton solution of IIB supergravity in section 2. An expanded discussion of the instanton action will be given in the appendix. Just as in the case of D-branes with $p>-1$ the classical supergravity solution [11] is singular at the origin, but the string theory description is expected to be well-defined at short distances [12, 13, 14, 15]. The fermionic zero modes of the IIB supergravity field theory in this background are obtained in section 3 by applying the generators of the sixteen broken supersymmetries to the instanton field configuration. The counterpart of these modes in the IIB superstring will be obtained in section 4 where the fermionic zero modes are given by attaching fermionic open string vertex operators to the boundary of world sheets. Integration over the sixteen fermionic moduli induces new interactions, just such as it does in conventional

\footnotetext{
${ }^{3}$ We are using the convention that the $\mathrm{R} \otimes \mathrm{R} q$-form potentials are denoted $C^{(q)}$.
} 
field theory 16$]$.

In section 5 we will discuss interactions that are induced at leading order in an instanton background in both the supergravity and the string descriptions. These include a $\lambda^{16}$ term and a $R^{4}$ term (where $R$ is the Riemann curvature). The latter is a one-instanton correction to the tree level [17, 18] and one loop 19] $R^{4}$ terms with the same tensor structure. These particular terms are non-stringy in origin since they can be deduced from IIB supergravity alone.

The explicit calculations of this paper are limited to a single unit-charged D-instanton background since little is known about multiply-charged D-instantons beyond the classical solutions of IIB supergravity. However, T-duality provides more detailed information. Thus, T-duality in nine dimensions (with euclidean time, $X^{0}$, compactified on a circle of circumference $l$ ) transforms the euclidean world-lines of D-particles to D-instantons. It is strongly believed that there are threshold bound states of $n$ D-particles for any integer $n$. The configuration in which the world-line of such a state is wrapped $m$ times has an action that is given by,

$$
\int_{0}^{2 \pi} d t\left(|n| e^{-\phi} \sqrt{g_{00}}+i n\left(\alpha^{\prime}\right)^{-1 / 2} C^{(1)} \frac{d X^{0}}{d t}\right)
$$

where $C^{(1)}$ is the $\mathrm{R} \otimes \mathrm{R}$ one-form potential $\left(0 \leq C^{(1)} \leq 2 \pi\right)$ and $g_{00}=l^{2}\left(d X^{0} / d t\right)^{2}\left(\alpha^{\prime}\right)^{-1}$ (where $l$ is the circumference of the compact euclidean time dimension). For the $m$-wrapped world-line $\left(\alpha^{\prime}\right)^{-1 / 2} \int d t\left(d X^{0} / d t\right)=2 \pi m$ and $\int d t\left(g_{00}\right)^{1 / 2}=2 \pi|m| l \alpha^{-1 / 2}$. Under T-duality $C^{(1)} \rightarrow C^{(0)}$ and $l e^{-\phi} \alpha^{-1 / 2} \rightarrow e^{-\phi}$. Thus, the action $S^{(m n)}$ for the D-instanton with charge $p=m n$ is,

$$
S^{(m n)}=-2 \pi i\left(m n C^{(0)}+i|m n| e^{-\phi}\right),
$$

which is equal to $-2 \pi i|m n| \tau$ for positive $m n$ (which we shall refer to as an instanton) and $2 \pi i|m n| \bar{\tau}$ for negative $m n$ (which we shall refer to as an anti-instanton), where $\tau=C^{(0)}+i e^{-\phi}$. Therefore there are multiply-charged D-instantons of charge $p=m n$ with degeneracy given by the number of partitions of $p$ into two integers. In section 6 we will use this information, together with known results of string perturbation theory, to motivate an $S L(2, Z)$ completion of the effective $R^{4}$ interaction of section 5 . We will see that for this to be correct there must be an interesting non-renormalization theorem that forbids perturbative corrections to this term beyond one loop in the zero-instanton sector - a property that has a plausible heuristic origin.

In section 7 we will consider an intrinsically stringy effect that arises from a world-sheet which is a disk with four massless closed-string tensor states attached. The fixed-angle scattering of gravitons on such a world-sheet is power behaved at high energy. This point-like behaviour arises, as in the bosonic theory, from the presence of massive closed-string states that couple to the boundary. However, although this is intriguing, its precise interpretation is unclear since integration over fermionic modes causes this process to vanish unless there are more external particles that are sources for the sixteen fermionic moduli. Furthermore, we have not taken into account possible effects of instantons with multiple charges that are required by the T-duality argument given above.

Beyond lowest order in the coupling constant scattering processes generically include connected world-sheets with more than one boundary, such as the annulus with both boundaries fixed at the same

\footnotetext{
${ }^{4}$ Thus, a D-particle wrapped in a positive direction is equivalent to an anti D-particle wrapped in a negative sense.
} 
space-time point. This is a loop of closed string - a loop correction to the D-instanton. The systematics of this perturbation theory involve cancellations between the Dirichlet theory divergences that are associated with the presence of the bosonic translation zero modes [20, 21]. These will also be outlined in section 7 . The boundary of moduli space that gives point-like scattering is not contained within the region that gives these cancelling divergences so that the puzzling point-like behaviour is not eliminated by cancellation of the Dirichlet divergences.

\section{Type IIB supergravity and the D-instanton}

The bosonic terms in the low-energy IIB supergravity are expressed in a manifestly $S L(2, R)$-invariant form in the Einstein frame,

$$
S=\frac{-1}{2 \kappa_{0}^{2}} \int d^{10} x \sqrt{-g}\left\{R-\frac{1}{2 \tau_{2}^{2}} \partial_{\mu} \tau \partial^{\mu} \bar{\tau}-\frac{1}{12 \tau_{2}}\left(\tau H_{N S}+H_{R}\right)_{\mu \nu \rho}\left(\bar{\tau} H_{N S}+H_{R}\right)^{\mu \nu \rho}\right\}+\cdots,
$$

where the fifth-rank field strength has been set to zero and we will set the arbitrary constant coupling to the value $\kappa_{0}=1$ for convenience. The terms represented by $\cdots$ in (3) include kinetic terms for the spin-1/2 complex Weyl fermion, $\lambda$, and the complex Weyl gravitino, $\psi_{\mu}$ (the 16-component spinor index is suppressed) and furthermore include an infinite series of terms of higher order in derivatives and higher fermion terms. The scalar and antisymmetric tensor fields arise in (3) in the combinations,

$$
\tau \equiv \tau_{1}+i \tau_{2}=C^{(0)}+i e^{-\phi}, \quad H=\left(\begin{array}{c}
H_{N S} \\
H_{R}
\end{array}\right),
$$

and $H_{N S}=d B_{N S}, H_{R}=d C^{(2)}$. The scalar fields parameterize the coset space, $S L(2, R) / U(1)$, in which the $U(1)$ represents a local symmetry acting on the fermions. Before choosing a gauge there are three scalar fields which enter the zweibein $V_{\alpha}^{ \pm}$,

$$
V=\frac{1}{\sqrt{-2 i \tau_{2}}}\left(\begin{array}{cc}
\bar{\tau} e^{-i \phi} & \tau e^{i \phi} \\
e^{-i \phi} & e^{i \phi}
\end{array}\right)
$$

where $0 \leq \phi \leq 2 \pi$. The local $U(1)$ rotations act from the right so that $V$ transforms into $V U(\alpha)$ (where $\left.U=\operatorname{diag}\left(e^{i \alpha}, e^{-i \alpha}\right)\right)$ which induces the shift, $\phi \rightarrow \phi-\alpha$. The global group $S L(2, R)$ acts from the left. Following the notation in 22] it is useful to define,

$$
P_{\mu} \equiv-\epsilon_{\alpha \beta} V_{+}^{\alpha} \partial_{\mu} V_{+}^{\beta}=i e^{2 i \phi} \frac{\partial_{\mu} \bar{\tau}}{2 \tau_{2}}, \quad Q_{\mu} \equiv-i \epsilon_{\alpha \beta} V_{+}^{\alpha} \partial_{\mu} V_{-}^{\beta}=\partial \phi-\frac{\partial_{\mu} \tau_{1}}{2 \tau_{2}},
$$

where $Q_{\mu}$ is a composite $U(1)$ potential that couples minimally to the fermions. The gravitino $\psi_{\mu}$ has $U(1)$ charge $1 / 2$ and the dialtino $\lambda$ has $U(1)$ charge $3 / 2$. The definitions (5) and (6) correspond to those given in 22] after performing the $S L(2, C)$ transformation that takes the IIB theory with the scalars living $S U(1,1) / U(1)$ coset to the one where the scalars parameterize a $S L(2, R) / U(1)$ coset. The gauge may be fixed by, for example, setting $\phi=0$, which will be used from here on. This means that generic $S L(2, R)$ transformations on the fields charged under $U(1)$ are associated with compensating $U(1)$ transformations and the global symmetry is nonlinearly realized. 
Perturbation theory in the zero-instanton sector is an expansion in fluctuations around constant values of the scalar fields, $\chi=\left\langle C^{(0)}\right\rangle$ and $\kappa=e^{-\langle\phi\rangle}$. Defining a complex coupling constant by,

$$
\tau_{0}=\chi+\frac{i}{\kappa}
$$

the fluctuating scalar fields can be expressed in a power series in $\kappa$ as,

$$
\tau \equiv C^{(0)}+i e^{-\phi}=C^{(0)}+\frac{i}{\kappa} e^{-\kappa \tilde{\phi}}=\tau_{0}+\tilde{C}^{(0)}-i \tilde{\phi}+\frac{i}{2} \kappa \tilde{\phi}^{2}+\cdots .
$$

where denotes a quantum fluctuation of a field.

The $N=2$ supersymmetries are $Q_{I}^{A}(I=1,2)$, where $Q_{1}^{A}$ and $Q_{2}^{A}$ are Majorana-Weyl spinors $(A=1, \cdots, 16)$ satisfying,

$$
\left\{\bar{Q}_{I}, Q_{J}\right\}=\delta_{I J} \gamma \cdot p
$$

In describing the instanton we will consider the complex combinations

$$
Q^{ \pm}=\frac{1}{2}\left(Q_{1} \pm i Q_{2}\right)
$$

Defining $\theta^{A}=Q^{-A}$ it follows that $Q^{+A}=(\gamma \cdot p \partial / \partial \theta)^{A}$. The euclidean continuation of $Q^{+}$annihilates the instanton solution. The supersymmetry transformations of the fields can be found in [22]. In particular, the fermion transformations have the form,

$$
\delta \lambda=i \gamma^{\mu} P_{\mu} \epsilon^{*}+. ., \quad \delta \psi_{\mu}=D_{\mu} \epsilon+. .
$$

where the spinor $\epsilon$ is a complex Weyl Grassmann variable so that $\bar{\epsilon}=\epsilon^{*} \gamma^{0}$.

The instanton is a euclidean saddle point of the bosonic part of type IIB supergravity in which the two scalar fields have a nontrivial profile (and $H=0$ ). The solution 11] is one in which the non-constant part of the $\mathrm{R} \otimes \mathrm{R}$ scalar field $C^{(0)}$ is imaginary so that,

$$
\hat{\tau}_{1}=\hat{C}^{(0)}=\chi+i f(r),
$$

where $r=|x-y|, y^{\mu}$ is the position of the instanton, $\chi$ and $f$ are real and $f(r=\infty)=0$. The fact that the instanton is a euclidean solution of the BPS type is particularly clear if the theory is reexpressed in terms of an eight-form potential $C^{(8)}$ by means of a duality transformation. This procedure, which is implicit in 11], is described in detail in the appendix.

As usual, the BPS condition implies that half of the euclidean supersymmetries, $Q^{+}$, annihilate the fields. In other words, setting $\epsilon^{*}=0$ in (11) it turns out that $\delta \lambda^{*}=0$ and $\delta \psi=0$. The first of these conditions follows directly from,

$$
d \hat{\tau}_{2}=i d \hat{\tau}_{1}, \quad \text { or } \quad d e^{-\hat{\phi}}=-d f .
$$

The instanton solution has the form, $\hat{g}_{\mu \nu}=\eta_{\mu \nu}$ and $\partial^{2} e^{\hat{\phi}}=0$ for $x^{\mu} \neq y^{\mu}$ so that

$$
e^{\hat{\phi}} \equiv h(r)=\kappa+\frac{c}{r^{8}}
$$

\footnotetext{
${ }^{5}$ Classical values of fields will be denoted by $\mathrm{a}^{\wedge}$ in the following.
} 
where $\kappa=e^{\hat{\phi}(r=\infty)}$, and $c=3|q| / \pi^{3 / 2}$ which follows from the quantization condition for a D-instanton of charge $q$ and a euclidean seven-brane. It will later be useful to represent $h$ in momentum space by its Fourier transform,

$$
\tilde{h}(p)=\kappa \delta^{(10)}(p)+\frac{c}{p^{2}} .
$$

Such an instanton has an action $S^{(q)}=2 \pi|q| / \kappa$, as shown in the appendix.

The solution can be transformed to the string frame by replacing the flat Einstein-frame metric by the string metric, $g_{\mu \nu}^{(s)}=e^{\phi / 2} \eta_{\mu \nu}$. In the string frame the solution is a space-time Einstein-Rosen wormhole [1] which joins two universes that are related by the interchange $r \rightarrow(c / \kappa)^{1 / 4} / r$. Since $e^{\hat{\phi}}$ gets large in the neck the field theoretic solution is not valid and the string theory D-instanton will have to be used to account for short-distance physics.

Substituting the solution in (12) gives,

$$
\hat{\tau}_{1}=\hat{C}^{(0)}=\chi+\frac{i}{\kappa}-i e^{-\hat{\phi}}=\tau_{0}-i \hat{\tau}_{2},
$$

so that

$$
\overline{\hat{\tau}}=\tau_{0}-\frac{2 i}{h}, \quad \hat{\tau}=\tau_{0}
$$

and $\hat{\tau}-\hat{\bar{\tau}}=2 i / h$.

In order to satisfy the second BPS condition, $\delta \psi=0$, the spinor $\epsilon$ must be covariantly constant, which implies,

$$
\epsilon=j(r) \epsilon_{0}
$$

where $j(r)=(2 / h)^{1 / 4}$ and $\epsilon_{0}$ is a constant sixteen-component chiral spinor. The broken supersymmetries associated with the spinor $\epsilon^{*}$ will generate the instanton solutions carrying fermionic zero modes.

The instanton has the Minkowski-space interpretation of a tunnelling process in which the initial and final $\mathrm{R} \otimes \mathrm{R}$ scalar Noether charges differ by $q$ units, $q_{f}-q_{i}=q$, where

$$
q_{i}=\frac{1}{2} \int_{x^{0}=-\infty} d^{9} x e^{2 \hat{\phi}} \partial_{0} \hat{C}^{(0)}, \quad q_{f}=\frac{1}{2} \int_{x^{0}=\infty} d^{9} x e^{2 \hat{\phi}} \partial_{0} \hat{C}^{(0)}
$$

This uses the fact that the Noether current associated with the shift symmetry, $C^{(0)} \rightarrow C^{(0)}+b$, is $j_{\mu}^{N}=e^{2 \phi} \partial_{\mu} C^{(0)} / 2$. In general a charge $q$ instanton amplitude will be accompanied by a phase factor $e^{2 \pi i \chi q}$ where $0 \leq \chi \leq 2 \pi$ (as with the $\theta$ term in the case of Yang-Mills instantons). This is equivalent to introducing a surface term in the action,

$$
i \chi \oint_{\partial M_{\infty}} * j^{N}=i \chi \oint_{\partial_{M_{\infty}}} \frac{1}{2} e^{2 \phi} * d C^{(0)}=i \chi \oint_{\partial M_{\infty}} \frac{1}{2} F_{9}
$$

which is an integral over the nine-sphere at $r=\infty$ (the last expression uses the dual potential described in the appendix). Adding this term to the action gives a total action for a charge $q>0$ instanton equal to

$$
S^{(q)}=-2 \pi i|q| \tau_{0}=2 \pi|q|\left(\frac{1}{\kappa}-i \chi\right)
$$


and for a charge $q<0$ anti-instanton equal to

$$
S^{(q)}=2 \pi i|q| \bar{\tau}_{0}=2 \pi|q|\left(\frac{1}{\kappa}+i \chi\right) .
$$

With $q=1$ this is precisely the same as the action obtained by expanding the theory in small fluctuations around the constant $\tau=\tau_{0}$ background in the presence of a source term in the action,

$$
S_{\text {source }}=-2 \pi i \int d^{10} x \tau(x) \delta^{(10)}(x-y)=-2 \pi i \tau_{0} .
$$

This is the D-instanton action that is the $p=-1$ case of the general D-brane action.

The classical values of the fields that enter into the supergravity are given by

$$
\hat{P}_{\mu}=-\frac{\partial_{\mu} h}{h}, \quad \hat{Q}_{\mu}=-\frac{i}{2} \frac{\partial_{\mu} h}{h}, \quad \hat{P}_{\mu}^{*}=0,
$$

so that $\hat{P}^{*}$ is not the complex conjugate of $\hat{P}^{\mu}$ since $\hat{\tau}_{1}$ is complex in the solution (17). The momentumspace expression for $\hat{P}^{\mu}$ has a pole,

$$
\tilde{\hat{P}}^{\mu}=\frac{2 i}{\kappa} \frac{c p^{\mu}}{p^{2}}+\cdots,
$$

where the dots indicate non-pole terms. Only the pole term, which depends on long-range effects, will enter the lowest-order on-shell amplitude calculations below.

As a simple example to illustrate the effect of the instanton background we will first consider the terms quadratic in the antisymmetric tensor fields. In the vacuum of the zero instanton sector the scalar field is constant, $\tau=\tau_{0}$, and these terms have the form,

$$
S_{H H}^{(0)}=\frac{\kappa}{24} \int d^{10} x\left(\tau_{0} H_{N S}+H_{R}\right)\left(\bar{\tau}_{0} H_{N S}+H_{R}\right)=\frac{\kappa}{24} \int d^{10} x G_{0} \bar{G}_{0},
$$

where

$$
G_{0}=\tau_{0} H_{N S}+H_{R}, \quad \bar{G}_{0}=\bar{\tau}_{0} H_{N S}+H_{R}
$$

(these correspond to the $G$ and $\bar{G}$ defined in 22] with the scalar fields equal to constant vacuum values).

In the one-instanton sector the quadratic terms in the fluctuations around the one-instanton background are obtained by substituting the expression for the instanton configuration of the scalar fields into the action (3). For example, for the antisymmetric tensor fields the result of substituting the classical solutions $\hat{C}^{(0)}$ and $\hat{\phi}$ into (3) is

$$
S_{H H}^{(1)}=-2 \pi i \tau_{0}+S_{H H}^{(0)}+S_{H H}^{\prime}+\cdots,
$$

where $\cdots$ indicates higher-order terms and terms involving fluctuations of the scalar fields, and

$$
\begin{aligned}
S_{H H}^{\prime} & =\frac{1}{24} \int d^{10} x \frac{c}{r^{8}}\left(\tau_{0} H_{N S}+H_{R}\right)\left(\tau_{0} H_{N S}+H_{R}\right) \\
& =\frac{1}{24} \int d^{10} x \frac{c}{r^{8}} G_{0} G_{0} .
\end{aligned}
$$

The action (28) changes by $2 \pi i \alpha$ under real shifts $\tau_{0} \rightarrow \tau_{0}+\alpha$ so that the effective action will have the expected factor of $e^{2 i \pi \tau_{0}}$ which is invariant under integer shifts of the background $\hat{C}^{(0)}$. The nonlocal term (29) agrees precisely with the description of the D-instanton as the source, (23), which has a non-zero contraction with the tree-level three-point couplings of two antisymmetric tensors. 


\section{Zero modes in a SUGRA instanton background.}

The bosonic zero modes are parameterized by the coordinates $y^{\mu}$ corresponding to the position of the D-instanton. Integration over $y^{\mu}$ enforces momentum conservation and results in a factor of $1 / \kappa^{5}$ (by a very similar argument to the one that results in a factor of $1 / g^{8}$ in the measure for the zero mode integral in the background of a Yang-Mills instanton [16]).

The fermionic zero modes can be determined, as usual, by applying the broken supersymmetry generators to the scalar solutions. The transformations in 22] can be adapted to the present problem by making the identification $\epsilon^{*}=j(x) \epsilon_{0}$ (where $j(x)$ is defined in (18)) and setting $\epsilon=0$. Since $\epsilon_{0}$ has 16 components this gives the possibility of $2^{16}$ independent separate instanton configurations obtained by applying the broken supersymmetries to the solution $\hat{P}_{\mu}$. The following are the terms involving up to eight powers of $\epsilon^{*}$,

$$
\begin{aligned}
& \hat{\lambda}=i \gamma^{\mu} \hat{P}_{\mu} \epsilon^{*}, \quad \hat{G}_{\mu \nu \rho}=3 D_{[\mu} \bar{\epsilon} \gamma_{\nu \rho]} \hat{\lambda} \\
& \hat{\psi}_{\mu}=\frac{1}{96}\left(\gamma_{\mu}^{\nu \rho \lambda} \hat{G}_{\nu \rho \lambda}-9 \gamma^{\rho \lambda} \hat{G}_{\mu \rho \lambda}\right) \epsilon^{*}, \quad \hat{F}_{\mu \nu \rho \lambda \sigma}^{5}=5 D_{[\mu} \bar{\epsilon} \gamma_{\nu \rho \lambda} \hat{\psi}_{\sigma]} \\
& \hat{e}_{\mu}^{r}=-i \bar{\epsilon} \gamma^{r} \hat{\psi}_{\mu}, \quad \hat{\psi}_{\mu}^{*}=-\frac{1}{480} i \gamma^{\rho_{1} \cdots \rho_{5}} \gamma_{\mu} \epsilon^{*} \hat{F}_{\rho_{1} \cdots \rho_{5}} \\
& \hat{G}_{\mu \nu \rho}^{*}=12 i \partial_{[\mu} \bar{\epsilon} \gamma_{\nu} \hat{\psi}_{\sigma]}^{*}, \quad \hat{\lambda}^{*}=\frac{1}{24} i \gamma^{\mu \nu \rho} \epsilon^{*} \hat{G}_{\mu \nu \rho}^{*}, \quad \hat{P}_{\mu}^{*}=D_{\mu} \bar{\epsilon}^{*} \hat{\lambda}^{*}
\end{aligned}
$$

(recall $\left.\bar{\epsilon}=\epsilon^{*} \gamma^{0}\right)$. The successive terms are defined iteratively in terms of $\hat{P}^{\mu}$. These zero modes are functions with momentum-space poles that can be seen by using (25).

In making contact with string theory we will be interested in physical fields satisfying the free equations of motion. In that case it is easy to see that terms with more than eight powers of $\epsilon^{*}$ in will not be needed. This is particularly clear in a light-cone parameterization in which the unphysical components of the fields are related to the $2^{8}$ physical components. The physical closed-string states can be packaged together into a light-cone scalar superfield, $\Phi(x, \theta)$, where $\theta^{a}(a=1, \cdots, 8)$ is an eight-component $S O(8)$ spinor, $\mathbf{8}_{\mathbf{s}}$, (and the inequivalent $S O(8)$ spinor $\mathbf{8}_{\mathbf{c}}$ will be represented by a dotted index). The equations of motion are imposed by requiring $\partial^{2} \Phi=0$. With this notation a 16-component chiral spinor has an $S O(8)$ decomposition,

$$
\epsilon^{* A} \rightarrow\left(\eta^{a}, \dot{\eta}^{\dot{a}}\right)
$$

$(A=1, \cdots, 16)$. The broken supersymmetries are generated by $\eta^{a} Q^{-a}+\dot{\eta}^{\dot{a}} Q^{-\dot{a}}$, where $Q^{-a}$ and $Q^{-\dot{a}}$ are the $S O(8)$ components of the broken supercharges and act on $\Phi$ by

$$
Q^{-a} \Phi=\sqrt{p^{+}} \theta^{a} \Phi, \quad Q^{-\dot{a}} \Phi=\left(\frac{\gamma^{i} p^{i}}{\sqrt{p^{+}}} \theta\right)^{\dot{a}} \Phi
$$

where $i=1, \cdots, 8$ labels the directions transverse to the light-cone. The classical fields, (30), are simply fields satisfying (at the linearised level)

$$
\hat{\Phi}=\left(\eta^{a} Q^{-a}+\dot{\eta}^{\dot{a}} Q^{-\dot{a}}\right) \hat{\Phi}
$$


Since the components of $Q^{-}$are linear in $\theta^{a}$ it is evident that a maximum of eight powers can be applied to $\Phi$, resulting in a maximum of eight powers of $\epsilon$.

The equations (30) define non-vanishing one-point functions for all components of a supermultiplet in the background of a single D-instanton. The Grassmann parameters are fermionic supermoduli corresponding to zero modes of $\lambda$ and must be integrated over together with the translational zero modes, $y^{\mu}$. A general Green function is given by an expression of the form,

$$
\begin{aligned}
& C \int d^{10} y d^{16} \epsilon_{0}\left\langle\Psi^{1}\left(x_{1}\right) \Psi^{2}\left(x_{2}\right) \cdots \Psi^{n}\left(x_{n}\right)\right\rangle_{\epsilon_{0}, y} \\
& =C \int d^{10} y d^{16} \epsilon_{0} \int D \Psi(x) \Psi^{1}\left(x_{1}\right) \Psi^{2}\left(x_{2}\right) \cdots \Psi^{n}\left(x_{n}\right) e^{-S^{(1)},}
\end{aligned}
$$

where $\Psi^{r}$ represents any of the fields of the theory and $S^{1}$ is the action in the one-instanton background which depends on $y$ through its dependence on the background $\hat{\tau}$ field configuration. We have not determined the overall constant, $C$, which can depend on $\kappa$ and will generalize to a function of $\tau$ and $\bar{\tau}$ when higher order fluctuations are considered. One way to pin down this dependence is to understand how the full effective action (including the sum over all instanton configurations) is invariant under $S L(2, Z)$. This will be discussed further in section 6 .

Since we will want to make comparisons with string theory we will define on-shell scattering amplitudes by the LSZ reduction that cancels the poles on the external legs in (34). In particular, the zero modes (30) define on-shell tadpoles in the presence of $s$ supermoduli,

$$
\langle\Psi\rangle_{s}=\zeta_{\Psi} \Delta^{-1} \hat{\Psi}_{s}
$$

where $\zeta_{\Psi}$ is the wave function for the on-shell closed-string state and $\Delta$ is its inverse propagator.

The leading contribution to the amplitude that follows from (34) is of the form

$$
e^{2 \pi i \tau_{0}} \int d^{10} y d^{16} \epsilon_{0}\langle\Psi\rangle_{s_{1}} \ldots\langle\Psi\rangle_{s_{n}}
$$

where $\sum_{i=1}^{n} s_{i}=16$.

\section{Zero modes in the stringy D-instanton background.}

The simplest open-string world-sheet that arises in a D-brane process is the disk diagram. In the case of the D-instanton the boundary satisfies Dirichlet conditions in all ten space-time directions. This means that there are no physical propagating open strings but there is an isolated open string supermultiplet consisting of a vector together with its spinor superpartner (23] and references therein). These remnants of the open-string sector are the zero-dimensional reduction of the ten-dimensional massless Yang-Mills supermultiplet. The vector field corresponds to the collective coordinate given by the instanton position,

$y^{\mu}$. Any world-sheet with vertex operators attached carrying momenta, $k_{i}^{\mu}$ has an overall factor of $\exp \left(i \sqrt{\kappa} \sum_{i} k_{i} \cdot y\right)$ and integration over $y^{\mu}$ leads to momentum conservation. The factors of $\sqrt{\kappa}$ in the open-string vertex lead to the factor of $\kappa^{-5}$ in the measure for the $y^{\mu}$ integration, as in the field theory. The open-string fermions give rise to the zero modes in the instanton background. Integration over the 
sixteen supermoduli leads to an overall factor of $\kappa^{8}$. In the complete string theory such factors of $\kappa$ will arise from powers of $\tau_{2}^{-1}$ and include the effects of dilaton couplings to the disk.

The disk with no states attached is defined by a functional integral that is simply a constant and is identified with the source term in the action, (23), and is equal to the action for a single D-instanton.

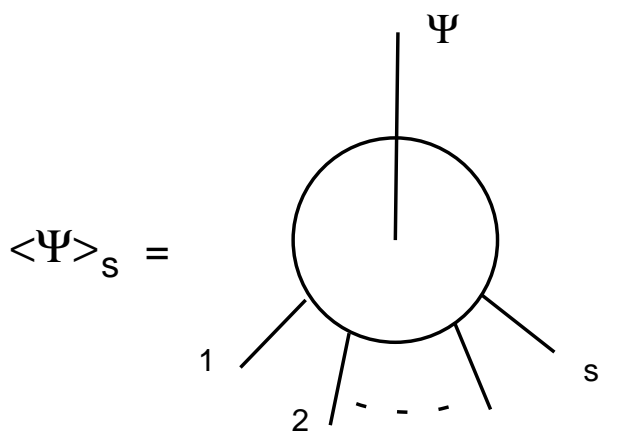

Fig. 1: An on-shell closed-string state, $\Psi$, coupling to $s$ open-string fermions on a disk with Dirichlet boundary conditions.

An instanton carrying some zero modes corresponds, at lowest order, to a disk world-sheet with open-string states attached to the boundary (as in fig. 1). The functional integration over the disk can be represented in an operator approach by transforming to a parameterization in which the world-sheet is a semi-infinite cylinder with a boundary end-state, $|B\rangle$. This state was constructed in 23] to preserve one half of the supersymmetries - both the world-sheet supersymmetries and the space-time supersymmetries. The formalism with manifest world-sheet supersymmetry requires two kinds of boundary state $-|B\rangle_{N S N S}$ and $|B\rangle_{R R}$ - in the NS $\otimes \mathrm{NS}$ and $\mathrm{R} \otimes \mathrm{R}$ sectors, respectively. These are related by the requirement that the boundary state preserve space-time supersymmetry. This is manifest in the formalism with manifest space-time supersymmetry formulated in a light-cone frame defined by choosing the axis of the cylinder to be the light-cone time, $X^{+}$. In that case there is a single boundary state that is annihilated by a complex combination of left-moving and right-moving space-time supercharges,

$$
Q^{+}|B\rangle \equiv(Q+i \tilde{Q})|B\rangle=0
$$

Enforcing this condition for all sixteen components of $Q^{+}$determines the boundary state, not just in the case of the D-instanton, but also for all the other $(p+1)$-instantons 泪. The other combination, $Q^{-}=Q-i \tilde{Q}$, which does not annihilate the boundary state is the broken supersymmetry. This lightcone gauge formalism should be derivable from a covariant supersymmetric formalism with world-volume $\kappa$-symmetry, such as in [24, 25, 26].

Applying a broken supersymmetry transformation $s$ times defines a state,

$$
|B\rangle_{s} \equiv \bar{\epsilon}_{0}^{A_{1}} Q^{-A_{1}} \cdots \bar{\epsilon}_{0}^{A_{s}} Q^{-A_{s}}|B\rangle,
$$

which applies in either the covariant formalism or the light-cone formalism In this notation a disk with one physical on-shell closed-string state attached to the interior and $s$ fermionic open strings attached to 
the boundary can be represented by

$$
\langle\Psi\rangle_{s}=\langle\Psi \mid B\rangle_{s}=\left\langle\delta^{s} \Psi \mid B\right\rangle,
$$

where $\delta^{s} \Psi$ is the variation of $\Psi$ obtained by applying $Q^{-} s$ times. Such tadpoles should be equal to the LSZ truncated fields of the supergravity theory (35). This is confirmed by direct calculation of the disk amplitudes, as follows.

In the light-cone gauge formalism the Grassmann spinor $\epsilon_{0}$ is written in terms of the $S O(8)$ spinors $\eta^{a}$ and $\dot{\eta}^{\dot{a}}$. The closed-string tadpoles are then given simply by the matrix elements,

$$
\langle\Psi\rangle_{s}=\left\langle\Psi\left|\eta^{a_{1}} Q^{-a_{1}} \cdots \eta^{a_{s}} Q^{-a_{s}}\right| B\right\rangle
$$

where each index $a_{1}, \ldots, a_{s}$ may be either undotted or dotted and contracted into the corresponding $S O(8)$ supercharge. It is easy to see that the resulting expressions are precisely those obtained from the field theory after LSZ reduction. The terms with purely undotted spinors package together into an $S O(8)$ superfield with components that are the $S O(8)$ physical light-cone gauge components of the $\langle\Psi\rangle_{s}$ of (35). The ground state of this supermultiplet is the complex scalar, $\left(i \zeta_{\phi}+\zeta_{C^{(0)}}\right)$, the linearized version of $\langle\tau\rangle$. The next state is $\zeta_{\lambda}^{a} \eta^{a}$, where $\zeta_{\lambda}^{a}$ is the complex wave function of the $S O(8)$ components of the spin- $1 / 2$ field, $\lambda^{a}=\lambda_{1}^{a}+i \lambda_{2}^{a}$. Continuing in this manner, all the components of the supermultiplet in (30) are reproduced.

These expressions can also be obtained covariantly by attaching a single closed-string vertex operator to the interior of the disk (which will be parameterized as the upper-half plane) together with fermionic open-string states attached to the boundary (the real axis). This may also be identified as the boundary state with $s$ fermionic open strings attached where the fermion vertex operator is given by,

$$
F_{-\frac{1}{2}}(x)=\bar{\epsilon}_{0} S(x) e^{-1 / 2 \phi(x)}, \quad F_{\frac{1}{2}}(x)=\bar{\epsilon}_{0} \gamma_{\mu} S(x) \partial X^{\mu}(x) e^{1 / 2 \phi(x)},
$$

in the superghost number $-1 / 2$ and $1 / 2$ pictures, respectively. The components of $\epsilon_{0}$ are the 16component spinor supermoduli that must be integrated in obtaining amplitudes. The total left-moving and right-moving superghost numbers on the disk must add up to -2 , which can be achieved by using combinations of vertices in appropriately chosen pictures.

With an even number of fermion open-string states attached to boundary the disk couples to closedstring bosons. The vertex operators in the -2 picture are

$$
\begin{aligned}
V^{N N} & =\zeta_{\mu \nu}^{N N} e^{-\phi} \psi^{\mu}(z) e^{-\tilde{\phi}} \tilde{\psi}^{\nu}(\bar{z}) e^{i k X} \\
V_{(n)}^{R R} & =\zeta_{(n)\left[\mu_{1} \cdots \mu_{n}\right]}^{R R} e^{-\phi / 2} \bar{S}(z) \Gamma^{\mu_{1} \ldots \mu_{n}} \tilde{S}(\bar{z}) e^{-3 \tilde{\phi} / 2} e^{i k X} .
\end{aligned}
$$

Here, $\zeta^{N N}$ is the wave function for the physical on-shell states in the NS $\otimes$ NS sector, $\phi, B_{N S}$ or $G$. The wave functions in the $\mathrm{R} \otimes \mathrm{R}$ sector, $\zeta_{(n)}^{R R}$, are $n$-form potentials describing $C^{(0)}, C^{(2)}$ and $C^{(4)}$. With this choice of pictures an even number of open-string operators can be coupled to the boundary of the disk with an equal number of $F_{+1 / 2}$ and $F_{-1 / 2}$ vertices.

The disk with an odd number of fermion states attached to the boundary couples to the closed-string fermionic states which are described by the vertex operators in the $-3 / 2$ picture,

$$
\begin{aligned}
V^{R N} & =\zeta_{\mu a}^{R N} e^{-\phi / 2} S^{a}\left(z_{2}\right) e^{-\tilde{\phi}} \tilde{\psi}^{\mu}\left(\bar{z}_{2}\right) e^{i k X} \\
\tilde{V}^{N R} & =\tilde{\zeta}_{\mu a}^{N R} e^{-\phi} \psi^{\mu}\left(z_{2}\right) e^{-\tilde{\phi} / 2} \tilde{S^{a}}\left(\overline{z_{2}}\right) e^{i k X} .
\end{aligned}
$$


Evidently, in order to give a total superghost number of -2 there must be a net superghost number of $-1 / 2$ from the open-string fermionic states attached to the boundary.

It is easy to evaluate the expectation values of a single closed-string vertex attached to the interior of a world-sheet with $s$ fermionic states attached to the boundary using standard techniques. Thus, for $s=0$ the only non-zero expectation values arise for the combination $\left\langle i V_{\phi}+V_{C^{(0)}}\right\rangle$, which is equal to $\langle\tau\rangle \sim\left(i \zeta_{\phi}+\zeta_{C}^{(0)}\right)$ as before.

The zero modes of the other fields in the supermultiplet are obtained by attaching fermionic openstring vertex operators (41) to the boundary. The expression for the disk with one fermionic open-string mode on the boundary and one closed-string fermion attached to the interior is given by

$$
\left\langle c F_{-\frac{1}{2}}(x) c \tilde{c} V^{R N}(z, \bar{z})\right\rangle
$$

This is evaluated making use of the boundary condition that reflects $\tilde{S}$ into $S$ and standard product expansions, giving,

$$
\langle\lambda\rangle_{1}=\left(\zeta_{a \mu}^{R N}+i \zeta_{a \mu}^{N R}\right) \gamma_{a b}^{\mu} \epsilon_{0}^{b} e^{i k y}=\bar{\zeta}_{\lambda} \epsilon_{0} e^{i k y}
$$

Where $\zeta_{\lambda}^{a}=\gamma_{a b}^{\mu}\left(\zeta_{\mu}^{b R N}+i \zeta_{\mu}^{b N R}\right)$ is the holomorphic combination of the two dilatinos. Attaching two fermionic zero modes to the boundary likewise gives non-vanishing one-point functions for a complex combination of the $\mathrm{NS} \otimes \mathrm{NS}$ and $\mathrm{R} \otimes \mathrm{R}$ two-forms, $B=B_{N S}+i C^{(2)}$. This comes from the correlation function,

$$
\begin{aligned}
\langle B\rangle_{2} & =\left\langle c F_{\frac{1}{2}}\left(x_{1}\right) \int d x_{2} F_{-\frac{1}{2}}\left(x_{2}\right) c \tilde{c}\left(V^{N N}(z, \bar{z})+i V_{(2)}^{R R}(z, \bar{z})\right\rangle\right. \\
& =\bar{\epsilon}_{0} \gamma^{\mu \nu \rho} \epsilon_{0} k_{[\mu}\left(\zeta_{\nu \rho]}^{N N}+i \zeta_{\nu \rho]}^{R R}\right) .
\end{aligned}
$$

With three fermionic zero modes attached the expression for the non-vanishing, holomorphic gravitino tadpole is

$$
\begin{aligned}
\langle\psi\rangle_{3} & =\left\langle c F_{\frac{1}{2}}(x) \int d x_{2} d x_{3} F_{-\frac{1}{2}}\left(x_{2}\right) F_{-\frac{1}{2}}\left(x_{3}\right) c \bar{c} V^{R N}(z, \bar{z})\right\rangle \\
& =\bar{\epsilon}_{0} \gamma^{\mu \nu \rho} \epsilon_{0} \bar{\zeta}_{\rho}^{N R} \gamma_{\mu} \epsilon_{0} k_{\nu}
\end{aligned}
$$

The disk with four fermion zero modes couples to the graviton and the self dual fourth-rank antisymmetric tensor with are both invariant under $S L(2, Z)$ S-duality of type IIB. The choice of pictures for the closed-string bosonic vertices in (43) requires that half the open-string fermions be in the $1 / 2$ picture and half in the $-1 / 2$ so that the tadpole is given by,

$$
\begin{aligned}
\langle h\rangle_{4} & =\left\langle c F_{1 / 2}\left(x_{1}\right) \int d x_{2} F\left(x_{2}\right)_{1 / 2} \int d x_{3} F_{-1 / 2}\left(x_{3}\right) \int d x_{4} F_{-1 / 2}\left(x_{4}\right) c \tilde{c} V^{N N}(z, \bar{z})\right\rangle \\
& =\bar{\epsilon}_{0} \gamma^{\rho \mu \tau} \epsilon_{0} \bar{\epsilon}_{0} \gamma^{\lambda \nu \tau} \epsilon_{0} \zeta_{\mu \nu} k_{\rho} k_{\lambda}
\end{aligned}
$$

which is the only covariant combination of four $\epsilon_{0}$ 's, two physical momenta and the physical polarization tensor.

Continuing in this manner it is clear that adding fermionic open-string states reconstructs the onepoint functions of the field theory in (30). All the terms with even numbers of fermionic moduli are 
formed from powers of the matrix,

$$
M^{\mu \nu}=\bar{\epsilon}_{0} \gamma^{\mu \nu \rho} \epsilon_{0} k_{\rho},
$$

which is the most general bilinear in two spinors and linear in the momentum. The fermionic terms, such as (49), have an extra factor of $\epsilon_{0}$.

\section{Lowest-order effective interactions}

The one-instanton terms in the field theory effective action can be deduced by considering on-shell amplitudes in the instanton background. The integration over the fermionic moduli soak up the sixteen independent fermionic zero modes. The contributions that arise at leading order in $\kappa$ are of the form (36) which is a product of the 'classical' fields, $\hat{\Psi}^{r}$ in (30) with a total of sixteen powers of $\epsilon_{0}$. This is the analogue of the leading term in the amplitude for gauge bosons in the Higgs-Yang-Mills instanton calculation in 27, which is again determined by the classical profile of the field. [0 In that case this particular kind of contact interaction is the first term in a series that reproduces an exponentially falling fixed-angle cross section with a scale that is symptomatic of the presence of solitonic states in the theory. An analogous interpretation of the contact term in IIB supergravity is to be expected.

The most obvious contact term is the one proportional to $\lambda^{16}$, which arises in IIB supergravity from the nonlocal Green function, defining $r_{m}=\left|x_{m}-y\right|$

$$
G_{\lambda^{16}}\left(\left\{r_{m}\right\}\right) \sim e^{2 \pi i \tau_{0}} \int d^{10} y \int d^{16} \epsilon_{0} \prod_{m=1}^{16}\left(\gamma_{\mu} \hat{P}^{\mu}\left(r_{m}\right) j\left(r_{m}\right) \epsilon_{0}\right)
$$

where we have not kept track of overall constant factors. This integral is well-defined because the function $P^{\mu}\left(r_{m}\right) j\left(r_{m}\right)$ is highly suppressed by the phase space volume at the origin and well-behaved at infinity. At long distances (52) looks like a $\lambda^{16}$ contact term. This is the term that can be obtained by use of the LSZ procedure described earlier and leads to a momentum-independent term in the S-matrix with sixteen external on-shell $\lambda$ particles proportional to,

$$
e^{2 \pi i \tau_{0}} \epsilon^{A_{1} \cdots A_{16}} \lambda^{A_{1}} \ldots \lambda^{A_{16}} .
$$

From the earlier discussion of the correspondence between the string theory and field theory zero modes it is evident that the same result is also obtained in string theory from diagrams with sixteen disconnected disks with a single $\lambda$ vertex operator and a single open-string fermion state attached to each one. The overall factor of $e^{2 \pi i \tau_{0}}$, which is characteristic of the stringy D-instanton [20, 21], is evaluated at $\chi=\operatorname{Re} \tau_{0}=0$ in the string calculation. In a more complete treatment the exponent should become $2 \pi i \tau$, which would include interactions due to fluctuations of $\phi$ and $C^{(0)}$. These should be deduced in a systematic manner by attaching vertex operators for these fields to further disconnected disks. String theory also contains further diagrams that have no direct analogue in field theory, in which more than one closed-string state is attached to each disk. Such diagrams contain the field theory contributions due to tree-level interactions in the instanton background but also contain intrinsically stringy effects, some of which will be described later.

\footnotetext{
${ }^{6}$ We are very grateful to Steven Shenker for pointing out this reference.
} 
We now turn to consider amplitudes with four external gravitons. The leading term in the field theory is again one in which each graviton is associated with four fermionic zero modes, which gives a Green function,

$$
G_{h^{4}}\left(\left\{r_{m}\right\}\right) \sim e^{2 \pi i \tau_{0}} \int d^{10} y \int d^{16} \epsilon_{0} \prod_{r=1}^{4}\left(\bar{\epsilon}_{0} \gamma^{\mu_{r} \sigma_{r} \rho} \epsilon_{0} \bar{\epsilon}_{0} \gamma_{\rho}^{\nu_{r} \tau_{r}} \epsilon_{0} k_{r}^{\left(\sigma_{r}\right.} \hat{P}_{r}^{\left.\tau_{r}\right)} j^{4}\left(r_{r}\right)\right) .
$$
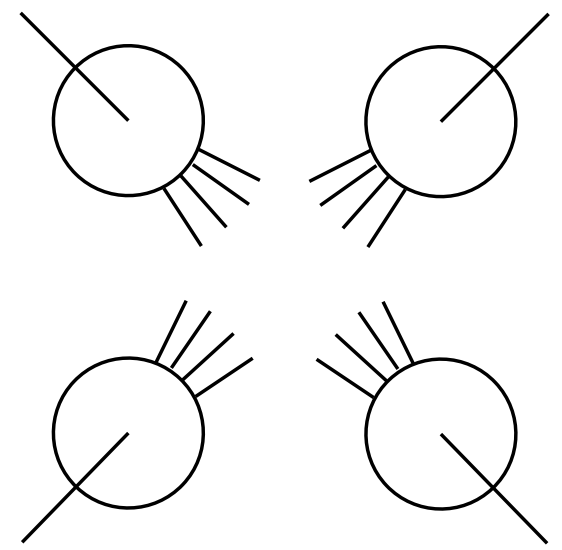

Fig. 2: The leading contribution to the scattering of four gravitons in the D-instanton background. The sixteen fermionic open strings represent the supermoduli that must be integrated. All disks have boundaries fixed at the same space-time point.

Integration over $y^{\mu}$ generates a nonlocal four-graviton interaction. Again, contact with the string calculation is made by considering the on-shell amplitude obtained by lopping off the external poles in momentum space, making use of (25), and contracting the free indices with the on-shell wave functions, $\zeta_{h}^{(r)}\left(k_{r}\right)$ which satisfy $k_{r \mu} \zeta^{(r) \mu \nu}=k_{r \nu} \zeta^{(r) \mu \nu}=0=k_{r}^{2}$. This corresponds to the string calculation in which the world-sheet (illustrated in fig. 2) consists of four disconnected disks to each of which is attached a single closed-string graviton vertex and four fermionic open-string vertices.

In both the string theory and the field theory the result is given as an integral of the product of four factors of $\langle h\rangle_{4}$ defined by (50),

$$
\begin{aligned}
A_{4}\left(\left\{\zeta_{h}^{(r)}\right\}\right) & =C \int d^{10} y d^{16} \epsilon_{0}\left\langle h^{(1)}\right\rangle_{4}\left\langle h^{(2)}\right\rangle_{4}\left\langle h^{(3)}\right\rangle_{4}\left\langle h^{(4)}\right\rangle_{4} e^{i \sqrt{\kappa} k_{r} \cdot y} \\
& =C \int d^{10} y d^{16} \epsilon_{0} \prod_{r=1}^{4}\left(\bar{\epsilon}_{0} \gamma^{\mu_{r} \sigma_{r} \rho} \epsilon_{0} \bar{\epsilon}_{0} \gamma^{\nu_{r} \tau_{r} \rho} \epsilon_{0} \zeta^{\left(\mu_{r}\right.} \tilde{\zeta}^{\left.\nu_{r}\right)} k_{r}^{\sigma_{r}} k_{r}^{\tau_{r}} e^{i \sqrt{\kappa} k_{r} \cdot y}\right),
\end{aligned}
$$

where the polarization tensor have been written as $\zeta^{\mu_{r} \nu_{r}}=\zeta^{\left(\mu_{r}\right.} \tilde{\zeta}^{\left.\nu_{r}\right)}$, which is sufficiently general for our purposes (and overall constants, including possible factors of $\kappa$, have been absorbed into $C$ ).

In order to evaluate the fermionic integrals we will choose a special frame in which $k_{r}^{+}=k_{r}^{-}=0$ and $k_{r} \cdot \zeta^{(r)}=k_{r} \cdot k_{r}=\zeta^{(r)} \cdot \tilde{\zeta}^{(r)}=0$ (which is possible for complexified momenta and polarizations). 
The spinor $\epsilon_{0}$ is naturally expressed in terms of its $S O(8)$ components so that

$$
\begin{aligned}
\langle h\rangle_{4} & =\left(-\eta_{a} \gamma_{a b}^{i j} \eta_{b} \dot{\eta}_{\dot{a}} \gamma_{\dot{a} \dot{b}}^{m n} \dot{\eta}_{\dot{b}}+\eta_{a} \gamma_{a \dot{b}}^{k i j} \dot{\eta}_{\dot{b}} \dot{\eta}_{\dot{a}} \gamma_{\dot{a} b}^{k m n} \eta_{b}\right) R_{i j m n} \\
& =-\frac{1}{2} \eta_{a} \gamma_{a b}^{i j} \eta_{b} \dot{\eta}_{\dot{a}} \gamma_{\dot{a} \dot{b}}^{m n} \dot{\eta}_{\dot{b}} R_{i j m n}
\end{aligned}
$$

where $R_{i j m n} \equiv k_{i} k_{m} \zeta_{(j} \tilde{\zeta}_{n)}$ is the linearized curvature and the second line follows from the first after a Fierz transformation.

The integral over the dotted and undotted spinors in (55) now factorizes and can be evaluated by using,

$$
\int d^{8} \eta^{a} \eta^{a_{1}} \cdots \eta^{a_{8}}=\epsilon^{a_{1} \cdots a_{8}}, \quad \int d^{8} \dot{\eta}^{\dot{a}} \dot{\eta}^{\dot{a}_{1}} \cdots \dot{\eta}^{\dot{a}_{8}}=\epsilon^{\dot{a}_{1} \cdots \dot{a}_{8}}
$$

Substituting in (55) the following tensors appear

$$
\begin{aligned}
& \epsilon_{a_{1} a_{2} \cdots a_{8}} \gamma_{a_{1} a_{2}}^{i_{1} j_{1}} \cdots \gamma_{a_{7} a_{8}}^{i_{4} j_{4}}=t^{i_{1} j_{1} \cdots i_{4} j_{4}}=\hat{t}^{i_{1} j_{1} \cdots i_{4} j_{4}}+\frac{1}{2} \epsilon^{i_{1} j_{1} \cdots j_{4} j_{4}} \\
& \epsilon_{\dot{a}_{1} \dot{a}_{2} \cdots \dot{a}_{8}} \gamma_{\dot{a}_{1} \dot{a}_{2}}^{i_{1} \dot{j}_{1}} \cdots \gamma_{\dot{a}_{7} \dot{a}_{8}}^{i_{4} j_{4}}=t^{i_{1} j_{1} \cdots i_{4} j_{4}}=\hat{t}^{i_{1} j_{1} \cdots i_{4} j_{4}}-\frac{1}{2} \epsilon^{i_{1} j_{1} \cdots j_{4} j_{4}}
\end{aligned}
$$

(where the notation follows 28]). There is an important sign difference between the eight-dimensional Levi-Cevita symbol in (58) and (59) which can be seen by a careful decomposition of $S O(9,1)$ into the two inequivalent spinor representations $\mathbf{8}_{\mathbf{c}}$ and $\mathbf{8}_{\mathbf{s}}$ of $S O(8)$. The result therefore contains two parityconserving terms

$$
\begin{aligned}
A_{4}\left(\left\{\zeta_{h}^{(r)}\right\}\right)= & C e^{2 i \pi \tau_{0}} \int d^{10} y e^{i \sum_{r} k_{r} \cdot y} \\
& \left(\hat{t}^{i_{1} j_{1} \cdots i_{4} j_{4}} \hat{t}_{m_{1} n_{1} \cdots m_{4} n_{4}}-\frac{1}{4} \epsilon^{i_{1} j_{1} \cdots j_{4} j_{4}} \epsilon_{m_{1} n_{1} \cdots m_{4} n_{4}}\right) R_{i_{1} j_{1}}^{m_{1} n_{1}} R_{i_{2} j_{2}}^{m_{2} n_{2}} R_{i_{3} j_{3}}^{m_{3} n_{3}} R_{i_{4} j_{4}}^{m_{4} n_{4}}
\end{aligned}
$$

It is notable that a parity-violating term, proportional to a single $\epsilon^{i_{1} j_{1} \cdots j_{4} j_{4}}$ tensor does not contribute. Although obtained in a manner that is not manifestly covariant these terms are easily covariantized by extending the indices to the ten-dimensional range. The term bilinear in the eight-dimensional LeviCevita tensor is the eight-dimensional Gauss-Bonnet term, which vanishes when the overall momentum is conserved. Since momentum conservation is only imposed in the one-instanton sector after integration over $y^{\mu}$ this term is non-zero for a fixed position of the instanton.

\section{An $S L(2, Z)$-invariant $R^{4}$ term and T-duality.}

The term in (60) bilinear in the tensor $\hat{t}$ has precisely the same form as terms that arise in the zero instanton sector that come both from the one-loop four-graviton amplitude [19] and from an $\left(\alpha^{\prime}\right)^{3}$ effect at tree level [18]. Collecting these three different contributions together in the Einstein frame gives an expression for the complete effective $R^{4}$ action that can be expressed in the form,

$$
S_{R^{4}}=\left(\alpha^{\prime}\right)^{-1}\left[a \zeta(3) \tau_{2}^{3 / 2}+b \tau_{2}^{-1 / 2}+c e^{2 \pi i \tau}+\cdots\right] R^{4} \equiv\left(\alpha^{\prime}\right)^{-1} f(\tau, \bar{\tau}) R^{4},
$$


where $R^{4}$ denotes the contractions $\hat{t} \hat{t} R^{4}$ in $(\sqrt[60]{60}$ ) and $\cdots$ indicates possible perturbative and nonperturbative corrections to the coefficient of $R^{4}$. The constants $a$ and $b$ given in the literature depend on the normalization conventions (such as the value of $\kappa_{0}$ in the action (3)), although their dependence on $\tau_{2}$ is as shown. For example, in the normalization given in [28], $a=3 \cdot 2^{-12}$ and $b=3 \cdot 2^{-18} \pi^{-5}$. Since we have not kept track of the factors of $\kappa$ the 'constant' $c$ has not been determined - it could, in principle, be a function of $\tau$ and $\bar{\tau}$.

However, the complete expression for $S_{R^{4}}$ must be invariant under $S L(2, Z)$ transformations, $\tau \rightarrow$ $(a \tau+b)(c \tau+d)^{-1}$ (with integer $a, b, c, d$, satisfying $a d-b c=1$ ), which provides very strong constraints on its structure. Since the $R^{4}$ factor is separately invariant the function $f(\tau, \bar{\tau})$ in $(61)$, must transform as a scalar under the $S L(2, Z)$ transformations. Such an expression necessarily involves a sum over all instanton and anti-instanton sectors. Although we have not derived the form of this sum there are some strong constraints which this term must satisfy:

- The tree-level perturbative contribution has the striking coefficient $\zeta(3)=\sum_{m>0}(1 / m)^{3}$.

- The one-loop perturbative term is of order $\tau_{2}^{-2}$ relative to this tree-level term. The non-vanishing of this one-loop term is possible only because the four external gravitons are just sufficient to soak up the eight fermionic zero modes on a toroidal world-sheet. Heuristically, the perturbative $R^{4}$ terms beyond one loop should vanish since extra fermionic zero modes on a higher genus surface require additional external states to give a non-vanishing contribution. Such a non-renormalization theorem has not appeared explicitly in the literature (as far as we know) and deserves further study.

- The multi-instanton contributions to the $R^{4}$ term can only come from a single instanton carrying multiple charge. Separated instantons carry extra fermionic zero modes 29 that lead to higher order derivative interactions.

- Such multiply-charged single D-instanton configurations are related by T-duality in nine euclidean dimensions to configurations in the type IIA theory in which the world-line of a single D-particle wraps $m$ times around the compact euclidean time dimension, $X^{0}$. This was shown in the introduction where it was argued that these instanton contributions are associated with a weight $e^{-S^{(m n)}}=e^{2 \pi i|m n| \tau}$ (and, correspondingly, $e^{-2 \pi i|m n| \bar{\tau}}$ for an anti-instanton). Furthermore, Tduality on a compactified $X^{9}$ direction relates the D-particle to the D-string. It then follows that these D-instanton configurations can be related by T-duality in eight euclidean dimensions to configurations of euclidean M-theory on $T^{3}$.

Rather tantalizingly, there is a simple function that satisfies all these criteria, namely,

$$
f(\tau, \bar{\tau})=T_{p n}^{-3} \equiv \sum_{(p, n) \neq(0,0)} \frac{\tau_{2}^{3 / 2}}{|p+n \tau|^{3}},
$$

where $\sum_{(p, n) \neq(0,0)}$ indicates the sum is over all positive and negative values of $p, n$ except $p=n=0$. It is easy to see that this function is invariant under $S L(2, Z)$ transformations. The expansion of this function for small $\tau_{2}^{-1}$ is given by first separating terms in the sum with $n=0$ and representing the rest 
as an integral,

$$
f=2 \zeta(3) \tau_{2}^{3 / 2}+\frac{\tau_{2}^{3 / 2}}{\Gamma(3 / 2)} \sum_{n \neq 0, p} \int_{0}^{\infty} d y y^{1 / 2} \exp \{-y(p+n \tau)(p+n \bar{\tau})\}
$$

Now the sum over $p$ should be reexpressed using the Poisson resummation formula,

$$
\sum_{p=-\infty}^{\infty} \exp \left(-\pi A(p+x)^{2}\right)=A^{-1 / 2} \sum_{m=-\infty}^{\infty} \exp \left(-\frac{\pi m^{2}}{A}+2 \pi i m x\right)
$$

which gives,

$$
f=2 \zeta(3) \tau_{2}^{3 / 2}+\frac{2 \pi^{2}}{3} \tau_{2}^{-1 / 2}+2 \tau_{2}^{3 / 2} \sum_{m, n \neq 0} \int_{0}^{\infty} d y \exp \left(-\frac{\pi^{2} m^{2}}{y}+2 \pi i m n \tau_{1}-y n^{2} \tau_{2}^{2}\right)
$$

where the second term accounts for the $m=0$ terms and we have used $\sum_{n} n^{-2}=\pi^{2} / 6$. The rest of the integral can either be evaluated by using a saddle point method or simply related to a $K_{1}$ Bessel function so that,

$$
\begin{aligned}
f(\tau, \bar{\tau})= & 2 \zeta(3) \tau_{2}^{3 / 2}+\frac{2 \pi^{2}}{3} \tau_{2}^{-1 / 2}+8 \pi \tau_{2}^{1 / 2} \sum_{m \neq 0 n \geq 1}\left|\frac{m}{n}\right| e^{2 \pi i m n \tau_{1}} K_{1}\left(2 \pi|m n| \tau_{2}\right) \\
= & 2 \zeta(3) \tau_{2}^{3 / 2}+\frac{2 \pi^{2}}{3} \tau_{2}^{-1 / 2} \\
& +4 \pi^{3 / 2} \sum_{m, n \geq 1}\left(\frac{m}{n^{3}}\right)^{1 / 2}\left(e^{2 \pi i m n \tau}+e^{-2 \pi i m n \bar{\tau}}\right)\left(1+\sum_{k=1}^{\infty}\left(4 \pi m n \tau_{2}\right)^{-k} \frac{\Gamma(k-1 / 2)}{\Gamma(-k-1 / 2)}\right),
\end{aligned}
$$

where we have used the asymptotic expansion for $K_{1}(z)$ for large $z$ in the second equation.

This expression incorporates all the points itemized earlier. It is an expansion with the appropriate perturbative terms (whose relative normalization differs from that in 28 where the definition of $\tau_{2}$ was different). The perturbative terms terminate after the one-loop term as suggested earlier. The nonperturbative terms have the form of a sum over single multiply-charged instantons and anti-instantons with action proportional to $|m n|$. If the original integer $p$ in (62) is identified with the discrete momentum (euclidean energy) of a compactified D-particle of charge $n$ then the Poisson resummation exchanges it for the winding number of the world-line and the result is that expected by T-duality from type IIA in nine dimensions. The terms in parenthesis in (66) represent the infinite sequence of perturbative corrections around the instantons of charge $m n$. Such corrections, beginning with the $\tau_{2}$-independent term, ought to be obtainable directly from the D-instanton calculation which would give an important check of the form of $f$. Thus, although we have by no means proven that the conjectured expression for $f$ in (62) is the $S L(2, Z)$-invariant coefficient of the $R^{4}$ term it satisfies several stringent constraints.

In pursuing possible connections with M-theory it should be of significance that the function $f$ is the $s=3 / 2$ case of an expression of the form

$$
f_{s}(\tau, \bar{\tau})=\zeta_{\Delta}(s)=\sum_{(p, n) \neq(0,0)}\left(\lambda_{p, n}\right)^{-s}
$$


where $\lambda_{p, n}$ are the eigenvalues of the laplacian on a two-torus. Explicitly, the laplacian on a two-torus with modular parameters $\tau_{1}$ and $\tau_{2}$ has eigenfunctions

$$
\psi_{p, n}(x, y)=\exp \left\{2 \pi i n x+2 \pi i y\left(\frac{p}{\tau_{2}}+\frac{n \tau_{1}}{\tau_{2}}\right)\right\},
$$

which satisfy the eigenvalue equation,

$$
\Delta \psi_{p, n}=\left(\partial_{x}^{2}+\partial_{y}^{2}\right) \psi_{p, n}=-\frac{4 \pi^{2}}{\tau_{2}^{2}}|p+n \tau|^{2} \psi_{p, n} \equiv \lambda_{p, n} \psi_{p, n} .
$$

The integers, $p$ and $n$, are Kaluza-Klein momenta which are interpreted as the discrete energy and charge of a $D$-particle compactified to nine euclidean dimensions. The function $f_{s}$ is a generalized Eisenstein series defined in [30, 31] by

$$
\begin{aligned}
E^{*}(\tau, \bar{\tau}, s) & =\frac{1}{2} \pi^{-s} \Gamma(s) f_{s}(\tau, \bar{\tau}) \\
& =\frac{1}{2} \pi^{-s} \Gamma(s) \sum_{(p, n) \neq(0,0)}\left(\frac{\tau_{2}}{|p+n \tau|^{2}}\right)^{s} \\
& =\pi^{-s} \Gamma(s) \zeta(2 s) E(\tau, \bar{\tau}, s)
\end{aligned}
$$

where $\zeta(s)$ is Riemann's zeta function,

$$
E(\tau, \bar{\tau}, s)=\frac{1}{2} \sum_{\left(l_{1} \mid l_{2}\right)=1}\left(\frac{\tau_{2}}{\left|l_{1} \tau+l_{2}\right|^{2}}\right)^{s},
$$

and $l_{1}$ and $l_{2}$ are relatively prime. One possibly significant property of these functions is that they satisfy the eigenvalue equation,

$$
\tau_{2}^{2}\left(\partial_{\tau_{1}}^{2}+\partial_{\tau_{2}}^{2}\right) E^{*}(\tau, \bar{\tau}, s)=s(s-1) E^{*}(\tau, \bar{\tau}, s)
$$

\section{$7 \quad$ Fixed-angle scattering.}

The leading contribution to the scattering amplitude for four closed-string states in the one-instanton sector is the term considered above in which each external graviton is attached to a separate disk (fig. 2), thereby generating the $R^{4}$ contact term. As we saw, the presence of this term can also be deduced within type IIB supergravity and there is nothing intrinsically stringy about it. This contact term grows like a positive power of the energy and should not be interpreted as a sign of fundamental point-like structure. Such terms arise also in the analogous calculation of high energy scattering in the presence of Yang-Mills instantons [27]. There they are the leading terms term in a power series expansion of a function that decreases exponentially with energy at fixed angle.

In this section we will consider higher-order processes, in which two or more closed-string states are attached to a disk but, for simplicity, we will not attach fermionic open-string states to the boundary. Such processes would obviously vanish after integration over the fermionic modes unless there are other

\footnotetext{
${ }^{7}$ We are grateful to Greg Moore and Richard Borcherds for pointing these references out to us.
} 
external states to act as sources for the sixteen fermionic moduli, so they should really be considered to be sub-processes in amplitudes with more external states (the analogues of terms in the action in a oneinstanton background). We will here simply evaluate such diagrams without performing the integrations over the fermionic moduli.

Diagrams in which there are two vertex operators attached to a single disk lead to contributions that are of order $\kappa$. For example, the coupling of two massless tensor states to a single disk with no external fermions is easily evaluated by considering $\left\langle V^{N N} V^{N N}\right\rangle,\left\langle V^{R R} V^{R R}\right\rangle$ and $\left\langle V^{N N} V^{R R}\right\rangle$. These quantities are readily evaluated and the result is that the two-graviton diagram vanishes 32 (as it does in the bosonic theory [21]). However, the process with two antisymmetric tensors is precisely the same as that deduced from the action (29).

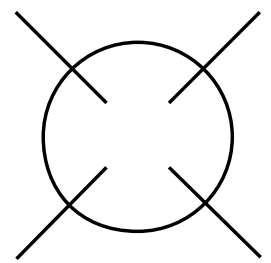

Fig. 3: Four closed-string states attached to a disk with Dirichlet boundary conditions. Since there are no fermionic moduli this diagram is a sub-process in a complete amplitude.

The diagram in fig. 3, is the lowest-order diagram with four gravitons that has no open-string states attached. Even though this only gives a non-zero contribution to the S-matrix in the presence of sources for the fermionic moduli it is instructive to analyze its behaviour in isolation since it illustrates explicitly the point-like effects of Dirichlet boundary conditions. Furthermore, with general external states this diagram also contains the open-string divergences characteristic of Dirichlet boundary conditions. These arise when a bosonic intermediate open string degenerates [6] and are guaranteed to cancel when all diagrams of a given order in $\kappa$-including disconnected diagrams - are added correctly [20, as we will review below.

In evaluating the diagram in fig. 3 there are many equivalent ways of assigning ghost numbers to the vertices in order to ensure that the total superghost number on the disk is -2 but it will be simplest to choose all the vertices to be in the $(0,0)$ picture apart from the vertex at $z_{1}=i$, which will be taken to be in the $(-1,-1)$ picture. 8 The amplitude is given by,

$$
A_{4 h}=\int d y_{2} d^{2} z_{3} d^{2} z_{4}\left\langle c \bar{c} V^{N N}\left(\zeta^{(1)}, z_{1}\right)(c+\bar{c}) V_{(0,0)}^{N N}\left(\zeta^{(2)}, i y_{2}\right) V_{(0,0)}^{N N}\left(\zeta^{(3)}, z_{3}\right) V_{(0,0)}^{N N}\left(\zeta^{(4)}, z_{4}\right)\right\rangle,
$$

where the coordinates of the vertices, $z_{r}=x_{r}+i y_{r}$, span the upper-half plane and we have fixed the Möbius symmetry by locating one vertex at $z_{1}=i$ and a second on the imaginary axis so that $z_{2}=i y_{2}$ $(0 \leq y \leq 1)$. The vertex in the $(-1,-1)$ picture is given by (42) and the vertex in the $(0,0)$ picture is given by

$$
V_{(0,0)}^{N N}=\zeta_{\mu \nu}\left(\partial X^{\mu}+i k_{\rho} \psi^{\rho} \psi^{\mu}\right)\left(\bar{\partial} X^{\nu}+i k_{\lambda} \bar{\psi}^{\lambda} \bar{\psi}^{\nu}\right) e^{i k X(z)}
$$

\footnotetext{
${ }^{8}$ In fact, it is interesting to see how the picture changing symmetry is preserved in this situation by the presence of boundary terms that are required in order to ensure the antisymmetric tensor gauge invariance 33 .
} 
Recall that we are considering this diagram to be a sub-process in which other external physical states may provide a total momentum $q$ (which could also be taken to vanish). After integrating over the position of the instanton the total momentum is conserved, $\sum_{r=1}^{4} k_{r}^{\mu}+q^{\mu}=0$, so that $s+t+u+q^{2}=0$, where $s=-\left(k_{1}+k_{2}\right)^{2}, t=-\left(k_{1}+k_{4}\right)^{2}$ and $u=\left(k_{1}+k_{3}\right)^{2}$. We shall consider a limit in which $s, t$ and $u$ are all large, which includes the 'fixed-angle' limit in which $q^{2}$ is small and

$$
s \rightarrow \infty, \quad t \rightarrow-\infty, \quad \frac{t}{s}=\frac{1}{2}(\cos \theta-1),
$$

where the scattering angle, $\theta$, is fixed. In open-string theories with Neumann boundary conditions as well as conventional closed-string theories the behaviour of the string amplitude in this limit is extremely soft [8, 9, 10] - the amplitude falls off exponentially as a function of $s, A(s, \theta) \sim \exp (-C s)$ where the constant $C$ depends on $\theta$ and on the genus of the world-sheet but not on the details of the string theory considered. This stringy behavior is also characteristic of scattering from D-branes with $p \geq 0$ in perturbation theory [32, 34 where the process is dominated by the cloud of open strings surrounding the brane. The presence of a Dirichlet boundary condition in the time direction leads to point-like scattering in the bosonic theory [5]. We will see that this is also true for the D-instanton of the type IIB superstring. The same is true for any of the $(p+1)$-instantons no matter how many compactified Neumann directions there are.

The amplitude (74) has an integrand that is the product of an assortment of prefactors that multiply a universal momentum-dependent factor,

$$
\exp (E)=\exp \left\{\sum_{i \neq j} k^{i} \cdot k^{j} \ln \left|\frac{z_{i}-z_{j}}{z_{i}-\bar{z}_{j}}\right|\right\},
$$

that depends on the scalar Green function on the half plane, with coordinates satisfying Dirichlet boundary conditions. The fact that amplitudes decrease as a power of the invariants in the asymptotic limits such as (76) is determined by the form of (77) although the prefactors will need to be considered in order to determine the precise power. The factor $e^{E}$ is exponentially suppressed at high energy and fixed angle unless the all but one of the $z_{i}$ approach the boundary of the disk. In that case the exponent vanishes. Using Möbius symmetry to fix one vertex at the origin this means that high-energy fixed-angle behaviour is dominated by the boundary of moduli space in which the other three vertex operators approach the boundary. In the case of Neumann boundary conditions the exponent in 77 is replaced by $\sum_{i \neq j} k^{i} \cdot k^{j} \ln \left|\left(z_{i}-z_{j}\right)\left(z_{i}-\bar{z}_{j}\right)\right|$ which never vanishes so that the amplitude is always exponentially suppressed.

The relevant boundary of moduli space that dominates in the fixed-angle limit is $y_{i} \rightarrow 0$. Expanding the terms in (77) around $y_{i}=0$ gives

$$
\begin{aligned}
& \ln \left|\frac{z_{1}-z_{i}}{z_{1}-\bar{z}_{i}}\right|^{2}=-\frac{4 y_{i}}{1+x_{i}^{2}}+O\left(y_{i}^{2}\right) ; \quad i=2,3,4 \\
& \ln \left|\frac{z_{i}-z_{j}}{z_{i}-\bar{z}_{j}}\right|^{2}=O\left(y_{i}^{2}\right) ; \quad i, j=2,3,4,
\end{aligned}
$$

where it has been assumed that $y_{i} \ll\left|x_{i}-x_{j}\right|$. Hence the factor $e^{E}$ behaves as

$$
\exp \left(E\left(s, t, q^{2} ; y_{2}, y_{3}, y_{4}, x_{3}, x_{4}\right)\right) \sim \exp \left\{4 s y_{2}+\frac{4 u}{x_{3}^{2}+1} y_{3}+\frac{4 t}{x_{4}^{2}+1} y_{4}+O\left(y_{i}^{2}\right)\right\} \text {. }
$$


It is easiest to analyse the asymptotic behaviour in the 'deep euclidean' limit in which $s, t$ and $u \rightarrow-\infty$ (so that $q^{2} \rightarrow+\infty$ ) since the exponent is positive definite in this kinematical region. In that case the integral is dominated by the region in which $y_{2} \sim s^{-1}, y_{3} \sim u^{-1}$ and $y_{4} \sim t^{-1}$. Thus, integrating with constant measure gives a behaviour $\sim(s t u)^{-1}$.

This power behaviour should also be recovered in any limit with large $|s|,|t|$ and $|u|$. These more general asymptotic limits can be analyzed explicitly by analytically continuing the $s, t, u$ invariants from a region in which the expression (80) is well-defined. We shall consider the example of the limit in (76) where $q^{2}$ is fixed. It is convenient to use the conformal transformation,

$$
e^{\tau+i \sigma}=\frac{i-z}{i+z}
$$

to map the upper-half plane onto the semi-infinite cylinder with the boundary at $\tau=0$. The factor (77) reduces to

$$
\exp \left\{-s \tau_{2}-t \tau_{3}-u \tau_{4}+O\left(\tau^{2}\right)\right\},
$$

where $-\infty<\tau_{i}<0$. If we now continue $s, t \rightarrow-\infty$ (implicitly letting $u \rightarrow+\infty$ ) the exponent becomes

$$
\exp \left\{-s\left(\tau_{2}-\tau_{4}\right)-t\left(\tau_{3}-\tau_{4}\right)+q^{2} \tau_{4}+O\left(\tau^{2}\right)\right\},
$$

which is convergent if $\tau_{2}<\tau_{4}$ and $\tau_{3}<\tau_{3}$. Since $\tau$ corresponds to the proper time along the cylinder this picks out a specific ordering of the three vertex operators along the cylinder, where $\tau_{4}$ is closest to the boundary. If the integration over $\tau_{i}$ is carried out ignoring the fact that there are other prefactors in the full expression for the amplitude this ordering gives a contribution of $\left(s t q^{2}\right)^{-1}$. The higher terms in the expansion are exponentially suppressed in this limit. For other $\tau$ orderings the integral is defined by taking other limits of $s, t$ and $u$. These are the orderings in which the vertex operators 2 and 3 are closest to the boundary which corresponds to $u, t \rightarrow-\infty$ (with $s \rightarrow \infty$ ) and $s, u \rightarrow-\infty$ (with $t \rightarrow \infty$ ), respectively. Adding all three contributions together (still ignoring the effect of prefactors in the integrand) gives a total factor proportional to

$$
\frac{1}{q^{2}}\left(\frac{1}{s t}+\frac{1}{t u}+\frac{1}{s u}\right)=\frac{s+t+u}{q^{2} s t u}=-\frac{1}{s t u} .
$$

As anticipated, this is the same asymptotic behaviour as in the limit $s, t, u \rightarrow-\infty$.

This is the essence of the analysis of the fixed-angle behaviour but the prefactors in (74) contribute powers of the invariants which depend on which particular particles are scattered and upon their helicity states. To begin with we will consider terms in the amplitude that involve contractions between all the external polarization tensors in a cyclic order, i.e., terms with prefactors of the form $\zeta_{\mu_{1} \mu_{2}}^{(1)} \zeta^{(2) \mu_{2} \mu_{3}} \zeta_{\mu_{3} \mu_{4}}^{(3)} \zeta^{(4) \mu_{4} \mu_{1}}$. It has previously been argued [6, 7] that such terms are particularly simple because the divergences associated with degenerations of intermediate open-string world-sheets do not contribute. Terms of this form will be referred to as 'cyclic' contractions. Obviously, other kinds of terms, such as those in which momenta contract into the polarization tensors arise in the complete process and will be considered later. 


\subsection{Cyclic contractions.}

In addition to the fact that there are three inequivalent cyclic contractions corresponding to the cyclically inequivalent permutations of the external states each of these consists of sixteen different terms since each polarization tensor can be contracted into its neighbour on either of two indices. Writing $\zeta_{\mu \nu}^{(r)}=\zeta_{\mu}^{(r)} \tilde{\zeta}_{\nu}^{(r)}$ the terms contributing to the $(1,2,3,4)$ cycle have kinematic prefactors of the form,

$$
\begin{gathered}
K(1, \overline{2} ; 2, \overline{3} ; 3, \overline{4} ; 4, \overline{1})=\zeta^{(1)} \cdot \tilde{\zeta}^{(2)} \zeta^{(2)} \cdot \tilde{\zeta}^{(3)} \zeta^{(3)} \cdot \tilde{\zeta}^{(4)} \zeta^{(4)} \cdot \tilde{\zeta}^{(1)} \\
K(1,2 ; \overline{2}, \overline{3} ; 3,4 ; \overline{4}, \overline{1})=\zeta^{(1)} \cdot \zeta^{(2)} \tilde{\zeta}^{(2)} \cdot \tilde{\zeta}^{(3)} \zeta^{(3)} \cdot \zeta^{(4)} \tilde{\zeta}^{(4)} \cdot \tilde{\zeta}^{(1)} \\
K(1, \overline{2} ; 2,3 ; \overline{3}, \overline{4} ; 4, \overline{1})=\zeta^{(1)} \cdot \tilde{\zeta}^{(2)} \zeta^{(2)} \cdot \zeta^{(3)} \tilde{\zeta}^{(3)} \cdot \tilde{\zeta}^{(4)} \zeta^{(4)} \cdot \tilde{\zeta}^{(1)} \\
\ldots \ldots
\end{gathered}
$$

If the external wave functions have no particular symmetry each of these prefactors multiplies an independent amplitude. However, if the $r$ th external particle is a graviton then the amplitude is symmetric under the interchange $r \leftrightarrow \bar{r}$ while the amplitude is antisymmetric under this interchange if the $r$ th particle is an antisymmetric tensor state. The resulting term in the amplitude with a prefactor of the form of the first line in 85 ) is proportional to

$$
\int d y_{2} d^{2} z_{3} d^{2} z_{4} \frac{K(1, \overline{2} ; 2, \overline{3} ; 3, \overline{4} ; 4, \overline{1}) k_{2} \cdot k_{4}\left(1-y_{2}^{2}\right)}{\left(i y_{2}+z_{4}\right)\left(i+i y_{2}\right)\left(i y_{2}-\bar{z}_{3}\right)^{2}\left(z_{3}-\bar{z}_{4}\right)^{2}\left(z_{4}+i\right)} e^{E} .
$$

The presence of inverse powers of world-sheet coordinates in the integrand alters the asymptotic fixed-angle behaviour that was deduced from the integral of $e^{E}$ with constant measure. The integral is still dominated by the end-point of the integration in which three vertex operators approach the boundary: $y_{2}, y_{3}, y_{4} \sim 0$. In this limit the denominators in (86) are finite at generic values of $x_{3}$ and $x_{4}$ and the power behaviour of the amplitude is enhanced from (84) to $(s t)^{-1}$ by the factor of $k_{2} \cdot k_{4}=-u / 2$ in the numerator. The end-points in moduli space at which two vertices approach each other on the worldsheet boundary (such as $z_{3} \rightarrow \bar{z}_{4}$ ) require special consideration because these are the regions in which the denominators in (86) vanish. It is precisely these boundaries of the integration region that can give rise to the novel Dirichlet logarithmic divergences illustrated in [20, 21] which should cancel with corresponding divergences arising from the degeneration limit of the annulus. However, these divergences only couple in channels with scalar quantum numbers so they do not arise for the parts of the amplitude involving cyclic contractions, which is the main reason for choosing to analyze these terms first.

If all the external states are gravitons the Dirichlet divergences are, in fact, absent from the noncyclic, as well as the cyclic, contractions. The behaviour of the full amplitude can be deduced from the term (86) together with Bose symmetrization and gauge invariance. For this reason the four-graviton amplitude will be considered first.

\section{Four external gravitons}

The amplitude with external gravitons is obtained by identifying $\tilde{\zeta}^{(r)}$ with $\zeta^{(r)}$ so that all sixteen terms for the ordering $(1,2,3,4)$ must be added with equal weight. This symmetrization leads to cancellations so that the leading power behaviour of the process is suppressed. 
These cancellations are well illustrated by comparing the contractions,

$$
\zeta_{\mu_{1}}^{(1)} \zeta_{\mu_{2}}^{(2)} \tilde{\zeta}_{\nu_{2}}^{(2)} \tilde{\zeta}_{\nu_{3}}^{(3)} k_{\rho_{2}}^{2} k_{\rho_{4}}^{4}\left\langle\psi^{\mu_{1}}\left(z_{1}\right) \tilde{\psi}^{\nu_{2}}\left(\tilde{z}_{2}\right)\right\rangle\left\langle\partial X^{\mu_{2}}\left(z_{2}\right) \bar{\partial} X^{\nu_{3}}\left(\bar{z}_{3}\right)\right\rangle\left\langle\tilde{\psi}^{\rho_{2}}\left(\bar{z}_{2}\right) \psi^{\rho_{4}}\left(z_{4}\right)\right\rangle,
$$

that arise in the calculation of the amplitude with the factor obtained by interchanging $\zeta_{\mu_{2}}^{(2)}$ with $\tilde{\zeta}_{\nu_{2}}^{(2)}$ and $z_{2}$ with $\bar{z}_{2}$

$$
\zeta_{\mu_{1}}^{(1)} \zeta_{\mu_{2}}^{(2)} \tilde{\zeta}_{\nu_{2}}^{(2)} \tilde{\zeta}_{\nu_{3}}^{(3)} k_{\rho_{2}}^{2} k_{\rho_{4}}^{4}\left\langle\psi^{\mu_{1}}\left(z_{1}\right) \psi^{\mu_{2}}\left(z_{2}\right)\right\rangle\left\langle\bar{\partial} X^{\nu_{2}}\left(\bar{z}_{2}\right) \bar{\partial} X^{\nu_{3}}\left(\bar{z}_{3}\right)\right\rangle\left\langle\psi^{\rho_{2}}\left(z_{2}\right) \psi^{\rho_{4}}\left(z_{4}\right)\right\rangle
$$

With $\tilde{\zeta}^{(2)}=\zeta^{(2)}$ then both these terms contribute a factor of $k^{2} \cdot k^{4} \zeta^{(1)} \cdot \tilde{\zeta}^{(2)} \zeta^{(2)} \cdot \tilde{\zeta}^{(3)}$ - but the interchange of arguments leads to a change of sign in the limit that $z_{2}$ is real. Thus, the leading behaviour derived from summing terms in the full amplitude that include these factors is suppressed relative to the individual terms.

Cancellations of this type and others that depend on the relative order of fermions must be systematically taken into account in adding the sixteen terms that make up one cyclic order. Summing the integrands of all sixteen terms of the form (86) in the limit of small $y_{2}, y_{3}$ and $y_{4}$ gives

$$
\frac{y_{2} y_{4}\left(1+x_{3} x_{4}\right)}{\left(1+x_{4}^{2}\right)^{2}\left(x_{4}-x_{3}\right)^{3} x_{3}^{3}} \exp (E)+O\left(y_{i}^{3}\right)
$$

which vanishes quadratically in the $y_{i}$ 's. This implies that the dominant contribution to the $(1,2,3,4)$ cyclic four graviton amplitude in the high-energy fixed-angle limit is proportional to

$$
\zeta^{(1)} \cdot \tilde{\zeta}^{(2)} \zeta^{(2)} \cdot \tilde{\zeta}^{(3)} \zeta^{(3)} \cdot \tilde{\zeta}^{(4)} \zeta^{(4)} \cdot \tilde{\zeta}^{(1)} \frac{1}{s^{2} t^{2}}
$$

where the $u^{-1}$ coming from (84) has cancelled the $k_{2} \cdot k_{4}$ in (86) and the extra inverse powers of invariants are produced by the $y_{2} y_{4}$ factor in (89). The other cyclically distinct contractions gives terms proportional to $s^{-2} u^{-2}$ and $t^{-2} u^{-2}$ respectively. There are many other terms that are suppressed by higher inverse powers of $s, t$ or $u$.

There is an apparent divergence in the coefficient of the leading power behaviour at the endpoint $x_{3}=x_{4}$ in (89). However, this is an illusion. The approximation used in (79) assumed that $\left|x_{i}-x_{j}\right| \gg y_{i}$ which is not true in the region that the vertices $i$ and $j$ approach each other and the boundary simultaneously. This is the boundary of moduli space in which an open-string strip pinches which may result in a logarithmic singularity associated with the level-one intermediate open-string states described earlier. But a simple scaling argument shows that there cannot be any divergences for the cyclic contractions with external gravitons. This may be seen by considering the limit in which the vertex operators 3 and 4 are close to each other and the boundary using the appropriately rescaled complex variable, $\rho_{4}$, defined by,

$$
z_{4}=x_{3}+y_{3} \rho_{4}
$$

The potential divergence arises as $y_{3} \rightarrow 0$. The measure $d^{2} z_{4} \rightarrow \int y_{3}^{2} d^{2} \rho_{4}$ so that divergences at the boundary come from terms which have $y_{3}^{-k}$ with $k \leq 3$. It is easy to see that the prefactor in (86) scales like $\epsilon^{-2}$ and hence there is no divergence in that limit.

When some or all of the external states are NS $\otimes$ NS antisymmetric tensors there are extra contributions to the amplitude. The leading term, of order $\kappa^{2}$, is now associated with a world-sheet that is 
the product of two disks with a pair of closed-string states attached to each. This contributes a term proportional to

$$
\int d^{10} y e^{-2 \pi / \kappa} A_{2 B}\left(k_{1}, k_{2} ; y\right) A_{2 B}\left(k_{3}, k_{4} ; y\right) e^{i q \cdot y},
$$

where $A_{2 B}\left(k_{1}, k_{2}, y\right)$ is the antisymmetric tensor two-point function and the $y^{\mu}$ integral gives $\delta^{(10)}\left(\sum_{r} k_{r}^{\mu}+\right.$ $q^{\mu}$ ), where $q^{\mu}$ is again the momentum arising from other particles in the process that soak up the sixteen fermionic zero modes. When the four antisymmetric tensors are attached to a single disk there are new issues related to the Dirichlet divergences that arise with non-cyclic contractions.

\subsection{Dirichlet divergences.}

Unlike with cyclic contractions, there are contributions to non-cyclic contractions in which there are scalar intermediate states present in some closed-string channels. For example, the contribution with kinematic coefficient $\zeta^{(1)} \cdot \tilde{\zeta}^{(2)} \zeta^{(2)} \cdot \tilde{\zeta}^{(1)} \zeta^{(3)} \cdot \tilde{\zeta}^{(4)} \zeta^{(4)} \cdot \tilde{\zeta}^{(3)}$ involves a set of of contractions analogous to $(74)$ but the structure of the prefactors is such that there is a logarithmic singularity from the integration region at which $z_{3}$ and $z_{4}$ approach each other and the world-sheet boundary simultaneously (this is made explicit by the rescaling in (91)). Such divergences, which are generic in D-instanton processes are guaranteed to cancel [20].

(a)

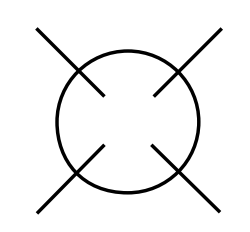

(b)

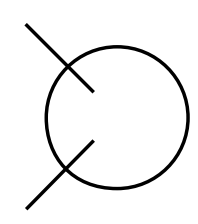

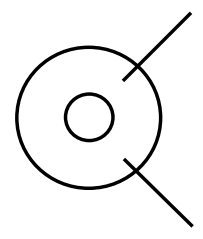

$\sim$
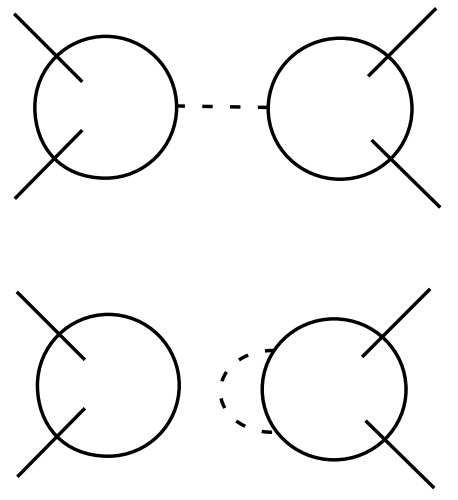

Fig. 4: World-sheets of order $\kappa^{3}$ with Dirichlet divergences due to open-string degenerations, indicated by dashed lines. (a) The divergence arising from the degeneration of the disk which is cancelled by (b), a single degeneration of the annulus. The double degeneration of the annulus is cancelled by other diagrams. All boundaries are mapped to the same space-time point.

For example, the divergence illustrated in fig. 4(a) corresponds to a factorization of the four-point function on the open string state and has the form

$$
\begin{gathered}
\left(k_{3}+k_{4}\right) \cdot\left(k_{1}+k_{2}\right) \int_{a}^{L} \frac{d q}{q} A_{2}\left(k_{1}, k_{2} ; y\right) A_{2}\left(k_{3}, k_{4} ; y\right) \\
=\ln (L / a) \frac{\partial}{\partial y^{\mu}} A_{2}\left(k_{1}, k_{2} ; y\right) \frac{\partial}{\partial y_{\mu}} A_{2}\left(k_{3}, k_{4} ; y\right),
\end{gathered}
$$


where $a$ is an arbitrary constant and $A_{2}$ is the two-point function on a disk for any of the massless closed-string states.

As expected [20, 21, the divergence cancels when account is taken of other (disconnected) diagrams of the same order in $\kappa$ but with more boundaries. In this example the relevant diagram is the product of an annulus and a disk with two states attached to each and all three boundaries mapped to the same point in the target space, $y^{\mu}$. The limit in which one open-string strip of the annulus degenerates again leads to a logarithmic divergence due to the intermediate level-one open-string vector state. This divergent term has the form,

$$
\frac{1}{2} \ln (L / b)\left\{\frac{\partial^{2}}{\partial y^{2}} A_{2}\left(k_{1}, k_{2} ; y\right) A_{2}\left(k_{3}, k_{4} ; y\right)+A_{2}\left(k_{1}, k_{2}, y\right) \frac{\partial^{2}}{\partial y^{2}} A_{2}\left(k_{3}, k_{4} ; y\right)\right\},
$$

where $b$ is an arbitrary constant (which need not necessarily be equal to $a$ ). Adding (93) and (94) give a total derivative in $y$ so the $L$-dependence cancels after integration over the collective coordinates and hence the sum of the terms is finite.

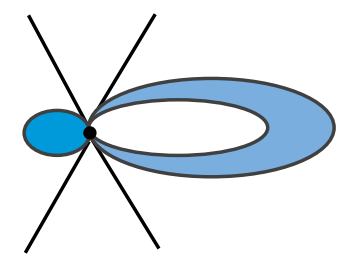

Fig. 5: The disconnected world-sheet in fig. 4(b) (a disk and an annulus with coincident boundaries) as seen from the space-time point of view and has the structure of a loop diagram correction to the instanton process.

It is noteworthy that the annular world-sheet in fig. 4(b) is really a kind of closed-string loop diagram from the target space point of view since all three boundaries coincide. The disconnected world-sheet of fig. 4(b) is shown from the space-time point of view in fig. 5, where it looks like a loop correction that is expected in the background of an instanton in field theory. Of course, from the field theoretic point of view such loops are ill-defined in ten dimensions. The cancellation of these divergences persists to all orders

The coefficient of $\ln a$ in (93) is of the same form as (92) apart from an overall factor of $\kappa s$. This suggests that in some more systematic treatment of the sum of diagrams these contributions could come be the first two terms in an expansion of

$$
e^{-2 \pi / \kappa} e^{2 \pi \kappa s \ln (b / a)}
$$

which is an exponentially suppressed contribution to the one-instanton amplitude.

These Dirichlet divergences arise from a boundary of moduli space that only includes part of the region which dominated in the fixed-angle limit. In other words, cancellation of the divergences does not eliminate the point-like behaviour. This is seen most clearly by considering the scattering process in a physical light-cone gauge, as follows.

Point-like scattering in the light-cone gauge

The point-like behaviour of scattering amplitudes on a disk is described in the light-cone gauge following 
the analysis in the bosonic string theory in [5]. Recall that tree-level elastic scattering of fundamental closed-string states can be described in a physical light-cone frame in which $\tau=X^{+}$, where $X^{+}$is time in the infinite momentum frame. In this parameterization the independent variables are, $X^{i}(i=1, \cdots, 8)$, the transverse coordinates. The scattering process may be mapped to the configuration in which the closed-string world-sheet has width in $\sigma$ equal to the total $p^{+}$carried by the incoming states, which is conserved throughout the process 35 .

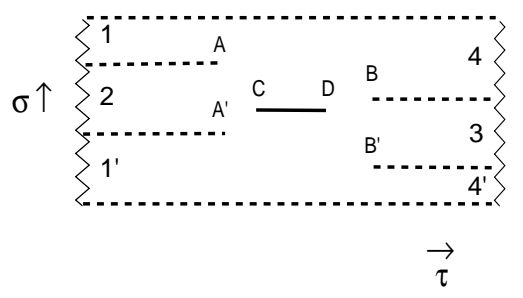

(a)

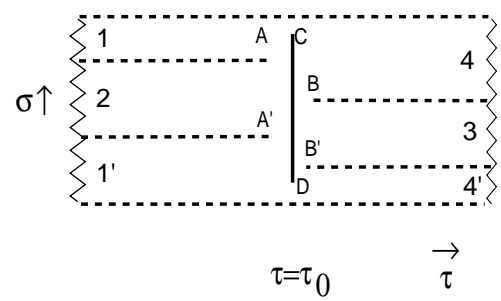

(b)

Fig. 6: The light-cone gauge parameterization of the world-sheets describing the scattering of four closed strings with (a) a Neumann boundary and (b) a Dirichlet boundary. Dashed horizontal lines are identified periodically to form closed strings.

In the original open-string theory (in which open strings had Neumann boundary conditions in all directions) the insertion of a single boundary in the world-sheet is a unitarity correction (fig. 5(a)), which is represented by the insertion of a boundary at fixed $\sigma$, on which the coordinates satisfy $\partial_{n} X^{i}=0$ (where $\partial_{n}$ indicates the normal derivative). The length of the boundary and its (complex) position are the moduli that are to be integrated. This describes an intermediate open string propagating in a closed-string process.

By contrast, the lowest-order contribution to the process in the presence of a D-instanton is represented by the insertion of a boundary with Dirichlet boundary conditions in all directions. This is mapped to a boundary at fixed $\tau=y^{+}$in the light-cone parameterization (fig. 5(b)). The boundary condition is now $\partial_{t} X^{i}=0$, or $X^{i}\left(\sigma, \tau=y^{+}\right)=y^{i}$. The length of this boundary and its (complex) position are again the three world-sheet moduli that are to be integrated over. Furthermore, the space-time position of the boundary, $y^{\mu}$, defines the translational zero modes of the instanton that form the bosonic moduli that are to be integrated over in performing the sum over all instanton configurations. The precise mapping involved is described in [5]. It applies equally well to any of the instantons that correspond to wrapping the euclidean world-volumes of $p$-branes around compactified directions. The difference in those cases is that $(p+1)$ of the $X^{i}$ satisfy Neumann conditions on the boundary and and there are only $(7-p)$ components to $y^{i}$.

This distinction between a unitarity correction and a D-instanton correction changes the high energy behaviour of scattering amplitudes. The fixed $\tau$ boundary in the case of the D-instanton represents a condition imposed on the sum over string histories that projects onto those histories which have a pointlike component at transverse position $y^{i}$ at the 'time' $\tau=y^{+}$. Fixed-angle scattering is dominated by those histories in which a finite fraction of the energy on the incoming strings is concentrated at a point at the moment that the strings scatter, leading to power-behaved amplitudes. The point-like fixed-angle 
behaviour comes from the integration region in which the points $A$ and $B$ in fig. 5(b) approach the vertical slit. This is independent of the Dirichlet divergence, described earlier, which arises from the end-point at which the vertical slit in fig. 5(b) spans the whole string so that the points $C$ and $D$ touch.

The point-like effects arising from the disk diagram are presumably resolved by accounting for other contributions to the amplitude. For example, in the calculation of a full amplitude there must be fermionic moduli provided by external sources. Furthermore, as we saw earlier, the presence of multiplycharged D-instantons is essential for the consistency of the theory. We have not considered how fixed-angle scattering might be affected by such contributions. Whereas the physics of D-particles is controlled by the eleven-dimensional Planck scale [13, 14, 15], the only scale that enters in the multi D-instanton moduli space is the ten-dimensional Planck scale [29] which is larger at weak coupling. It would be interesting to understand how this apparent distinction in scales is reflected in the physical properties of the theory.

Note: While this paper was being prepared several papers [36, 37, 38, 39] appeared in which related interesting issues concerning D-instantons are discussed.

\section{Acknowledgments:}

MBG is grateful to the Ecole Normale for hospitality in August 1996 where parts of this work were carried out.

\section{APPENDIX: The SUGRA instanton action}

The strategy is to transform the $\mathrm{R} \otimes \mathrm{R}$ scalar, $C^{(0)}$, to an eight-form potential, $C^{(8)}$, with field strength $F_{9}$. There are several arguments for using such a formulation of the IIB theory.f Firstly, the continuation from minkowskian to euclidian signature is very simple since the Maxwell-type lagrangian density, $F_{9}^{2}$, does not transform. Furthermore, the euclidean action is manifestly positive in this dual formulation, without the introduction of a boundary term. We will see that this implies the presence of a boundary term in the scalar field part of the usual type IIB euclidean action, which makes it positive.

In order to motivate the form of the dual action we start by considering the first-order minkowskian action (it will be sufficient to consider the scalar field part of the action with a flat metric since the metric is trivial in the instanton solution in the Einstein frame [11]),

$$
S\left(P^{(9)}, C^{(0)}\right)=\frac{1}{4} \int\left(d \phi \wedge * d \phi+e^{-2 \phi} P^{(9)} \wedge * P^{(9)}-2 C^{(0)} \wedge d P^{(9)}\right),
$$

where $P^{(9)}$ is a nine-form field. The functional integral is of the form $\int D P^{(9)} D C^{(0)} e^{i S}$ and we will write $S=S_{M}$ when the signature is minkowskian and $S=i S_{E}$ when it is euclidean.

Later we will see that performing the integration over $P^{(9)}$ first gives rise to the $C^{(0)}$ kinetic terms in the IIB action together with a specific surface term. But to begin with we will consider integrating over $C^{(0)}$ first, which imposes the constraint $d P^{(9)}=0$ that is solved by $P^{(9)}=d C^{(8)}$ (ignoring global

\footnotetext{
${ }^{9}$ Much of this appendix is based on discussions with Gary Gibbons
} 
issues). Substituting this in the action (96) gives,

$$
S\left(C^{(8)}\right)=\frac{1}{4} \int\left(d \phi \wedge * d \phi+e^{-2 \phi} d C^{(8)} \wedge * d C^{(8)}\right) .
$$

With Minkowski signature this can be expressed as

$$
S_{M}\left(C^{(8)}\right)=\frac{1}{4} \int\left(d \phi-e^{-\phi} * d C^{(8)}\right) \wedge *\left(d \phi+e^{-\phi} * d C^{(8)}\right)
$$

where we have made use of the properties of a $p$-form, $a^{(p)}$, under Poincaré duality in ten dimensions,

$$
* * a^{(p)}=(-1)^{p+1} a^{(p)} .
$$

With Euclidean signature Poincaré duality implies,

$$
* * a^{(p)}=(-1)^{p} a^{(p)},
$$

and the action $(97)$ becomes

$$
S_{E}\left(C^{(8)}\right)=\frac{1}{4} \int\left(d \phi \pm e^{-\phi} * d C^{(8)}\right) \wedge *\left(d \phi \pm e^{-\phi} * d C^{(8)}\right) \pm \frac{1}{2} \int e^{-\phi} d \phi \wedge d C^{(8)}
$$

The first term on the right-hand side is positive semi-definite while the second term is a total derivative that can be written as,

$$
\int e^{-\phi} d \phi \wedge d C^{(8)}=-\int d\left(e^{-\phi} d C^{(8)}\right)=-\oint_{\partial M} e^{-\phi} F_{9},
$$

where $F_{9}=d C^{(8)}$ and $\partial M=\partial M_{\infty}+\partial M_{0}$ denotes the boundary of space-time which consists of the $S^{9}$ at $r=\infty\left(\partial M_{\infty}\right)$ and the $S^{9}$ around $r=0\left(\partial M_{0}\right)$. From this it follows that the euclidean action satisfies a Bogomol'nyi bound,

$$
S_{E}\left(C^{(8)}\right) \geq \frac{1}{2}\left|\oint_{\partial M} e^{-\phi} F_{9}\right| .
$$

The action is minimized and the bound saturated when the first term in (101) vanishes, so that

$$
d \phi \pm e^{-\phi} * d C^{(8)}=0
$$

are satisfied by the BPS and anti-BPS solutions. This is the dual form of (13). The 'electric' charge on the instanton is given by the topological integral $\oint_{\partial M_{\infty}} F_{9} / 2=2 \pi q$, whereas in the original formulation it is equal to the integral of the Noether charge for the $C^{(0)}$ shift symmetry. This gives the instanton action,

$$
S^{(q)}=\frac{2 \pi}{\kappa}|q| .
$$

The parameter $q$ becomes quantized in integer units by the Dirac-Nepomechie-Teitelboim argument for a instanton in the presence of a euclidean seven-brane. 
In order to see how the euclidean IIB action arises from (96) we will now consider performing the $P^{(9)}$ integration first. This is obtained by writing the action as the quadratic form,

$$
\begin{aligned}
S_{E}\left(P^{(9)}, C^{(0)}\right)= & \frac{1}{4} \int\left(d \phi \wedge * d \phi+e^{-2 \phi}\left(P^{(9)}+i e^{2 \phi} * d C^{(0)}\right) \wedge *\left(P^{(9)}+i e^{2 \phi} * d C^{(0)}\right)\right. \\
& \left.+e^{2 \phi} d C^{(0)} \wedge * d C^{(0)}-2 d\left(i C^{(0)} P^{(9)}\right)\right),
\end{aligned}
$$

where the factors of $i$ arise from the Wick rotation of the first two terms on the right-hand side of (96) together with the fact that $S_{E}=-i S$. Shifting variables to $P^{\prime(9)}=\left(P^{(9)}+i e^{2 \phi} * d C^{(0)}\right)$ and performing the $P^{\prime(9)}$ integration in the functional integral gives the scalar field part of the IIB supergravity action

together with a surface term, $\oint_{\partial M} d\left(e^{2 \phi} C^{(0)} \wedge * d C^{(0)}\right)$. This shift of variables is only possible if $d C^{(0)}=i d f$ is imaginary, as anticipated earlier, in which case the action is given by,

$$
S_{E}(f)=\frac{1}{4} \int\left(\left(d \phi \wedge * d \phi-e^{2 \phi} d f \wedge * d f\right)-2 d\left(e^{2 \phi} f \wedge * d f\right)\right),
$$

which gives rise to equations of motion (13) with instanton solutions (14) [11] in which $f=A+e^{-\phi}$, where $A$ is an arbitrary constant. The action for a charge $q$ instanton, obtained by substituting the solution into (107), comes entirely from the surface term which gets contributions from $\partial M_{\infty}$ and $\partial M_{0}$. Using the explicit solution for $e^{\phi}$ the boundary term at $r=0$ can be shown to equal $2 \pi(A-1 / \kappa) q$ while that at $r=\infty$ is equal to $2 \pi A q$ so that the action is again given by (105). If we choose $A=0$ the action comes entirely from the surface at $r=\infty$.

The presence of the boundary term in the scalar action affects the functional integral in that it restricts the class of functions, $f$, to those that have continuous derivatives (in other words, the action is only additive for this class of functions). From the duality relation $F_{9} \sim e^{\phi} d f$ this is consistent with the fact that $F_{9}$ is continuous in the absence of seven-brane sources.

\section{References}

[1] K. Becker, M. Becker and A. Strominger, Fivebranes, membranes and nonperturbative string theory, hep-th/9507158, Nucl. Phys. B456 (1995) 130.

[2] H. Ooguri and C. Vafa, Summing up D instantons, HUTP-96-A036, hep-th/9608079.

[3] J.A. Harvey and G. Moore, Five-brane instantons and $R^{2}$ couplings in N=4 string theory, EFI-96-38, hep-th/9610237.

[4] M.B. Green and M. Gutperle, Light cone supersymmetry and D-branes, hep-th/9604091, Nucl. Phys. B476 (1996) 484.

[5] M.B. Green, Point-like structure and off-shell dual amplitudes, Nucl. Phys. B124 (1977) 461.

[6] M.B. Green, Space-time duality and Dirichlet string theory, Phys. Lett. B266 (1991) 325.

[7] M. Gutperle, Multiboundary effects in Dirichlet string theory, hep-th/9502106, Nucl. Phys. B444 (1995), 487. 
[8] G. Veneziano, Construction of a crossing symmetric, Regge behaved amplitude for linearly rising trajectories, Nuov. Cim. 57a (1968) 190.

[9] V. Allesandrini, D. Amati and B. Morel, The asymptotic behavior of the dual pomeron amplitude, Nuov. Cim. 7A (1971) 797.

[10] D.J. Gross and P.F. Mende, String theory beyond the Planck scale, Nucl. Phys. B303 (1988) 407.

[11] G.W. Gibbons, M.B. Green and M.J. Perry, Instantons and seven-branes in type IIB superstring theory, hep-th/9511080, Phys. Lett. B370 (1996) 37.

[12] E. Witten, Bound states of strings and p-branes, hep-th/9510135, Nucl. Phys. B460 (1996) 335.

[13] D. Kabat and P. Pouliot, A comment on zero-brane quantum mechanics, hep-th/9603127, Phys. Rev. Lett. 77 (1996) 1004.

[14] U.H. Danielsson, G. Ferretti, B. Sundborg, D particle dynamics and bound states, hep-th/9603081, Int. J. Mod. Phys. A11 (1996) 5463.

[15] M.R. Douglas, D. Kabat, P. Pouliot and S.H. Shenker, D-branes and short distances in string theory, RU-96-62, hep-th/9608024.

[16] G. t Hooft, Computation of the quantum effects due to a four-dimensional pseudoparticle, Phys. Rev. D14 (1976) 3432.

[17] M.T. Grisaru , A.E.M Van de Ven and D. Zanon, Two-dimensional supersymmetric sigma models on Ricci flat Kahler manifolds are not finite, Nucl. Phys. B277 (1986) 388 ; Four loop divergences for the $N=1$ supersymmetric nonlinear sigma model in two-dimensions; Nucl. phys. B277 (1986) 409.

[18] D.J. Gross and E. Witten, Superstring modifications of Einstein's equations Nucl. Phys. B277 (1986) 1.

[19] M.B. Green and J.H. Schwarz, Supersymmetric dual string theory III, Nucl. Phys. B198 (1982) 441.

[20] J. Polchinski, Combinatorics of boundaries in string theory, hep-th/9407031, Phys. Rev. D50 (1994) 6041.

[21] M.B. Green, A gas of D-instantons, Phys. Lett. B354 (1995); Boundary effects in string theory, contributed to STRINGS 95: Future Perspectives in String Theory, USC, March 1995, hep-th/9507121.

[22] J.H. Schwarz, Covariant field equations of chiral N=2 D=10 supergravity, Nucl. Phys. B226 (1983) 269.

[23] M.B. Green, Point-like states for type IIB superstrings, hep-th/9403040, Phys. Lett. B329 (1994) 435. 
[24] M. Cederwall, A. von Gussich, B.E.W. Nilsson and A. Westerberg, The Dirichlet super three brane in ten-dimensional type IIB supergravity, GOTEBORG-ITP-96-13, hep-th/9610148; M. Cederwall et al., The Dirichlet super p-branes in ten dimensional type IIA and type IIB supergravity, GOTEBORG-ITP-96-14, hep-th/9611159.

[25] M. Aganagic, C. Popescu and J.H. Schwarz, D-brane actions with local kappa symmetry, CALT68-2081A, hep-th/9610249; Gauge invariant and gauge fixed D-brane actions, CALT-68-2088, hepth/9612080

[26] E. Bergshoeff and P.K. Townsend, Super D-branes, DAMTP-R-96-53, hep-th/9611173.

[27] V.A. Rubakov and M.E. Shaposhnikov, Electroweak baryon number nonconservation in the early universe and in High-energy collisions, CERN-TH-96-13, hep-ph/9603208.

[28] A.A. Tseytlin, Heterotic Type I superstring duality and low energy effective action, hep-th/9512081, Nucl. Phys. B467 (1996) 383.

[29] M.B. Green and M. Gutperle, Configurations of two D-instantons, DAMTP-96-110, hep-th/9612127.

[30] D. Zagier, Eisenstein series and the Riemann zeta function in Tata Inst. Fund. Res. Studies in Math., 10, Tata Inst. Fundamental Res., Bombay, 1981.

[31] A. Terras, Harmonic analysis on symmetric spaces and applications I, Springer, New York-Berlin, 1985.

[32] I.R. Klebanov and L. Thorlacius, The size of p-branes, hep-th/9510200, Phys. Lett. B371 (1996) 51.

[33] M. Gutperle, Ph.D thesis, University of Cambridge, (unpublished).

[34] J.L.F. Barbon, D-brane form-factors at high energy, hep-th/9601098, Phys. Lett. B382 (1996) 60.

[35] S. Mandelstam, Interacting string picture of dual resonance models, Nucl. Phys. 64 (1973) 205.

[36] V. Periwal, Matrices on a point as the theory of everything, PUPT-1665, hep-th/9611103; Antibranes and crossing symmetry, PUPT-1676, hep-th/9612215.

[37] N. Ishibashi, H. Kawai,Y. Kitazawa and A. Tsuchiya, A large $N$ reduced Model as superstring, KEKTH-503, hep-th/9612115.

[38] A.A. Tseytlin, Type IIB instanton as a wave in twelve dimensions, CERN-TH-96-333, hepth/9612164.

[39] J.L.F. Barbon, Fermion exchange between D-instantons, CERN-TH-96-360, hep-th/9701075. 